\title{
Tandem RCM-isomerization-cyclopropanation reactions
}

| Álvaro Mallagaray, Gema Domínguez, Ana Gradillas and Javier PérezCastells*

Facultad de Farmacia, Dpto. Química, Universidad San Pablo CEU, Urb. Montepríncipe, ctra. Boadilla km 5,300 Boadilla del Monte, 28668 Madrid

jpercas@ceu.es

\section{General procedures:}

${ }^{1} \mathrm{H}$ NMR and ${ }^{13} \mathrm{C}$ NMR spectra were taken on a Bruker $\mathrm{AM}-300$ spectrometer. Chemical shifts $\left(^{\mathrm{TM}}\right)$ are in parts per million relative to tetramethylsilane at $0.00 \mathrm{ppm}$. IR spectra were determined on a Perkin-Elmer 780 spectrometer. TLC analyses were performed on commercial aluminium sheets bearing $0.25-\mathrm{mm}$ layer of Merck Silica gel $60 \mathrm{~F}_{254}$. Silica gel 60 Merk of 230-400 mesh ASTM was used for column chromatography. Medium pressure liquid chromatography (MPLC) was performed using Biotage Si 25+M 2593-2 compact columns. Elemental analyses were carried out at the Elemental Analysis Center of the Complutense University of Madrid, using a Perkin Elmer $2400 \mathrm{CHN}$. The solvents for the reactions carried under argon atmosphere were distilled just before their use. THF was refluxed over $\mathrm{Na/Benzophenone,} \mathrm{DCM}$ and toluene over $\mathrm{CaH}$. All reactives were bought to Aldrich and Across.

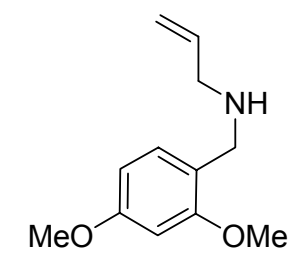

Preparation of $\mathbf{N}$-(2,4-dimethoxybenzyl)prop-2-en-1-amine. A solution of allylamine (3.4 g, $60.2 \mathrm{mmol}), 2,4$-dimethoxybenzaldehyde $(10.0 \mathrm{~g}, 84.2$ $\mathrm{mmol}$ ) and sodium triacetoxyborohydride (17.8 g, $84.2 \mathrm{mmol})$ in $210 \mathrm{~mL}$ of 1,2dichloroethane was stirred at room temperature for 24 h. $50 \mathrm{~mL}$ of $\mathrm{Na}_{2} \mathrm{CO}_{3}$ (10\%) were added and the 1,2-dichloroethane was eliminated under reduced pressure. The crude was extracted with diethyl ether $(3 \times 50 \mathrm{~mL})$, dried with $\mathrm{MgSO}_{4}$ and concentrated in vacuo. The resulting mixture was purified by flash 
chromatography (Hex:AcOEt 4:1/ $\mathrm{MeOH})$, affording $8.7 \mathrm{~g}(70 \%)$ as a pale yellow oil.

${ }^{1} \mathrm{H}-\mathrm{NMR}\left(\mathrm{CDCl}_{3}, 300 \mathrm{MHz}\right): \delta 1.68(\mathrm{~s}, 1 \mathrm{H}), 3.23\left(\mathrm{dd}, \mathrm{J}_{1}=4.4 \mathrm{~Hz}, \mathrm{~J}_{2}=1.6\right.$ $\mathrm{Hz}, 2 \mathrm{H}), 3.72(\mathrm{~s}, 2 \mathrm{H}), 3.81(\mathrm{~s}, 3 \mathrm{H}), 3.82(\mathrm{~s}, 3 \mathrm{H}), 5.08-5.22(\mathrm{~m}, 2 \mathrm{H}), 5.87-6.00$ $(\mathrm{m}, 1 \mathrm{H}), 6.44(\mathrm{~d}, \mathrm{~J}=7.7 \mathrm{~Hz}, 1 \mathrm{H}), 6.46(\mathrm{~s}, 1 \mathrm{H}), 7.13(\mathrm{~d}, \mathrm{~J}=7.7 \mathrm{~Hz}, 1 \mathrm{H}) .{ }^{13} \mathrm{C}-\mathrm{NMR}$ $\left(\mathrm{CDCl}_{3}, 75 \mathrm{MHz}\right): \delta 48.2,51.5,55.2,55.3,98.5,103.5,115.7,120.7,130.5$, 137.1, 158.6, 160.0. IR (film): v 3350, 3070, 3000, 2940, 2915, 1650, 1610, $1580 \mathrm{~cm}^{-1}$. Anal. Calcd. for $\mathrm{C}_{12} \mathrm{H}_{17} \mathrm{NO}_{2}$ : C. $69.54 ;$ H. 8.27; N. 6.76. Found: C. $69.41 ;$ H. 8.12; N. 6.58.

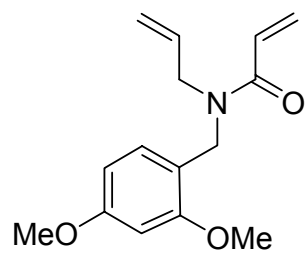

Preparation of $\quad \mathrm{N}$-(2,4-dimethoxybenzyl)-N-prop-2-en-1-ylprop-2enamide (8). 3-Chloropropionic acid chloride (1.2 g, $9.7 \mathrm{mmol})$ was added to a solution of $\mathrm{N}$-(2,4-dimethoxybenzyl)prop-2-en-1-amine $(2.0 \mathrm{~g}, 9.7 \mathrm{mmol})$ and triethylamine $(3.9 \mathrm{~g}, 38.6 \mathrm{mmol})$ in $50 \mathrm{~mL}$ of THF under argon at $0^{\circ} \mathrm{C}$. After 24 h. at room temperature the crude was filtered, washed with $\mathrm{NaHCO}_{3}(2 \times 20$ $\mathrm{mL})$, brine $(20 \mathrm{~mL})$, dried with $\mathrm{MgSO}_{4}$ and the solvent was eliminated under reduced pressure. The resulting mixture was purified by flash chromatography (Hex:AcOEt 9:1 to 1:1) affording $1.58 \mathrm{~g}(63 \%)$ of 8 as a pale yellow oil.

${ }^{1} \mathrm{H}-\mathrm{NMR}$ (DMSO-d $, 300 \mathrm{MHz}, 80^{\circ} \mathrm{C}$ ): $\delta 3.77$ (s, 3H), 3.80 (s, 3H), 3.95 (d, Eliminado: RMN $\mathrm{J}=3.7 \mathrm{~Hz}, 2 \mathrm{H}), 4.47(\mathrm{~s}, 2 \mathrm{H}), 5.06-5.13(\mathrm{~m}, 2 \mathrm{H}), 5.64(\mathrm{~d}, \mathrm{~J}=10.4 \mathrm{~Hz}, 1 \mathrm{H}), 5.70-$ $5.85(\mathrm{~m}, 1 \mathrm{H}), 6.15(\mathrm{~d}, \mathrm{~J}=16.5 \mathrm{~Hz}, 1 \mathrm{H}), 6.51(\mathrm{~d}, \mathrm{~J}=7.9 \mathrm{~Hz}, 1 \mathrm{H}), 6.58(\mathrm{~s}, 1 \mathrm{H})$, $6.70\left(\mathrm{dd}, \mathrm{J}_{1}=16.5 \mathrm{~Hz}, \mathrm{~J}_{2}=10.4 \mathrm{~Hz}, 1 \mathrm{H}\right), 7.02(\mathrm{~d}, \mathrm{~J}=7.3 \mathrm{~Hz}, 1 \mathrm{H}) .{ }^{13} \mathrm{C}-\mathrm{NMR}$ $\left(\mathrm{CDCl}_{3}, 75 \mathrm{MHz}\right), 2$ rotamers: $\delta 43.1,45.4,47.9,49.4,54.9,55.0,55.1,55.2$, 98.0, 98.3, 103.5, 104.0, 116.2, 116.8, 117.1, 117.8, 127.7, 127.8, 130.5, 132.8, 133.0, 157.8, 158.3, 160.0, 160.3, 166.5. IR (film): v 3060, 3000, 2920, 2820, 1740, 1640, 1620, $1580 \mathrm{~cm}^{-1}$. Anal. Calcd. for $\mathrm{C}_{15} \mathrm{H}_{19} \mathrm{NO}_{3}$ : C. 68.94; H. 7.33; N. 5.36. Found: C. 69.18 ; H. 7.56; N. 5.21. 
General procedure for the synthesis of amides by condensation. A solution of the corresponding acid $(1.0 \mathrm{mmol}), \mathrm{HOBT}(1.5 \mathrm{mmol})$ and DCC $(1.5$ $\mathrm{mmol}$ ) in DCM (5 mL) was stirred for one hour under argon. $\mathrm{N}-(2,4-$ dimethoxybenzyl)prop-2-en-1-amine $(1 \mathrm{mmol})$ was then added solved in DCM $(5 \mathrm{~mL})$ via canula and the mixture was stirred for $24 \mathrm{~h}$. The crude was filtered, washed with $\mathrm{HCl} 1 \mathrm{~N}(5 \mathrm{~mL})$, brine, dried with $\mathrm{MgSO}_{4}$ and the solvent was eliminated under reduced pressure. Finally the mixture was purified by flash chromatography.

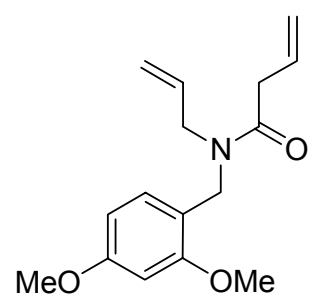

Preparation of $N$-(2,4-dimethoxybenzyl)-N-prop-2-en-1-ylbut-3enamide (1). Following the typical procedure for the synthesis of amides by condensation, the reaction of $\mathrm{N}$-(2,4-dimethoxybenzyl)prop-2-en-1-amine (14.5 $\mathrm{mmol})$ and vinylacetic acid (1.2 $\mathrm{g}, 14.5 \mathrm{mmol})$ afforded after flash chromatography (Hex:AcOEt 6:1) $3.2 \mathrm{~g}(81 \%)$ of 1 as a transparent oil.

${ }^{1} \mathrm{H}-\mathrm{NMR}\left(\mathrm{DMSO}-\mathrm{d}_{6}, 300 \mathrm{MHz}, 80^{\circ} \mathrm{C}\right.$ ): $\delta 3.18$ (d, J=5.5 Hz, 2H), 3.77 (s, $3 \mathrm{H}), 3.80(\mathrm{~s}, 3 \mathrm{H}), 3.88(\mathrm{~s}, 2 \mathrm{H}), 4.40(\mathrm{~s}, 2 \mathrm{H}), 5.00-5.12(\mathrm{~m}, 4 \mathrm{H}), 5.62-5.85(\mathrm{~m}$, $1 \mathrm{H}), 5.87-5.99(\mathrm{~m}, 1 \mathrm{H}), 6.51(\mathrm{~d}, \mathrm{~J}=6.7 \mathrm{~Hz}, 1 \mathrm{H}), 6.58(\mathrm{~s}, 1 \mathrm{H}), 7.02(\mathrm{~d}, \mathrm{~J}=6.7$, 1H). ${ }^{13} \mathrm{C}-\mathrm{NMR}\left(\mathrm{CDCl}_{3}, 75 \mathrm{MHz}\right), 2$ rotamers: $\delta 38.2,38.3,42.7,45.5,47.4,49.4$, 55.0, 55.1, 55.2, 55.2, 98.0, 98.4, 103.6, 104.0, 116.1, 116.7, 117.0, 117.4, $117.4,117.9,127.8,130.6,131.9,133.0,158.0,158.3,160.0,160.3,171.1$, 171.2. IR (film): v 3060, 3000, 2920, 2820, 1640, 1620, $1580 \mathrm{~cm}^{-1}$. Anal. Calcd. for $\mathrm{C}_{16} \mathrm{H}_{21} \mathrm{NO}_{3}$ : C. 69.79 ; H. 7.69; N. 5.09. Found: C. 70.01; H. 7.85; N. 5.30.<smiles>C=CCCC(=O)N(CC=C)Cc1ccc(OC)cc1OC</smiles> 
Preparation of $\quad N$-(2,4-dimethoxybenzyl)-N-prop-2-en-1-ylpent-4enamide (11). Following the typical procedure for the synthesis of amides by condensation, the reaction of $\mathrm{N}$-(2,4-dimethoxybenzyl)prop-2-en-1-amine (12.1 $\mathrm{mmol}$ ) and 4-pentenoic acid (1.4 g, $14.5 \mathrm{mmol})$ afforded after flash chromatography (Hex:AcOEt 6:1) $2.9 \mathrm{~g}(83 \%)$ of $\mathbf{1 1}$ as a transparent oil.

${ }^{1} \mathrm{H}-\mathrm{NMR}\left(\mathrm{DMSO}-\mathrm{d}_{6}, 300 \mathrm{MHz}, 80^{\circ} \mathrm{C}\right): \delta 2.29-2.33(\mathrm{~m}, 2 \mathrm{H}), 2.43-2.51(\mathrm{~m}$, Eliminado: RMN 2H), $3.77(\mathrm{~s}, 3 \mathrm{H}), 3.80(\mathrm{~s}, 3 \mathrm{H}), 3.88(\mathrm{~d}, \mathrm{~J}=4.9 \mathrm{~Hz}, 2 \mathrm{H}),, 4.41(\mathrm{~s}, 2 \mathrm{H}), 4.93-5.10$ (m, 4H), 5.63-5.98 (m, 2H), 6.51 (d, J= $7.3 \mathrm{~Hz}, 1 \mathrm{H}), 6.57$ (s, 1H), 6.99 (sa, 1H). ${ }^{13} \mathrm{C}-\mathrm{NMR}\left(\mathrm{CDCl}_{3}, 75 \mathrm{MHz}\right), 2$ rotamers: $<\delta 28.6,29.1,31.9,33.0,42.6,45.3$, $47.4,49.3,54.8,54.9,55.0,55.0,97.9,98.2,103.4,103.8,114.8,114.9,115.9$, 116.6, 116.6, 117.8, 127.6, 130.3, 132.8, 132.9, 137.4, 137.4, 157.8, 158.2, 159.8, 160.1, 172.4, 172.4. IR (film): $v 3350,3000,2915,2815,1630,1610$, $1580 \mathrm{~cm}^{-1}$. Anal. Calcd. for $\mathrm{C}_{17} \mathrm{H}_{23} \mathrm{NO}_{3}$ : C. 70.56 ; H. 8.01; N. 4.84. Found: C. 7.65; H. 7.88; N. 4.76.<smiles>COc1ccc(CN2CC=CC2=O)c(OC)c1</smiles>

Preparation of 1-(2,4-dimethoxybenzyl)-1,5-dihydro-2H-pyrrol-2-one (9). A solution of catalyst II $(24.3 \mathrm{mg}, 0.03 \mathrm{mmol})$ in $5 \mathrm{~mL}$ of dry toluene was added via cannula to a solution of amide $8(150.0 \mathrm{mg}, 0.6 \mathrm{mmol})$ in dry toluene ( $20 \mathrm{mM}$ ) under argon. After $3 \mathrm{~h}$ the resulting dark brown solution was filtered by Celite and the solvent was eliminated under reduced pressure. The resulting mixture was purified by flash chromatography (Hex:AcOEt 1:2), affording 128.5 $\mathrm{mg}(95 \%)$ of 9 as a yellow oil.

${ }^{1} \mathrm{H}-\mathrm{NMR}\left(\mathrm{CDCl}_{3}, 300 \mathrm{MHz}\right): \delta 3.79(\mathrm{~s}, 3 \mathrm{H}), 3.81(\mathrm{~s}, 3 \mathrm{H}), 3.89(\mathrm{~s}, 2 \mathrm{H}), 4.58$ (s, 2H), 6.15-6.18 (m, 1H), $6.43(\mathrm{~d}, \mathrm{~J}=8.5 \mathrm{~Hz}, 1 \mathrm{H}), 6.44(\mathrm{~s}, 1 \mathrm{H}), 7.00-7.02(\mathrm{~m}$, $1 \mathrm{H}), 7.12(\mathrm{~d}, \mathrm{~J}=8.5 \mathrm{~Hz}, 1 \mathrm{H}) .{ }^{13} \mathrm{C}-\mathrm{NMR}\left(\mathrm{CDCl}_{3}, 75 \mathrm{MHz}\right): \delta 40.1,52.7,55.3$, 55.3, 98.3, 104.1, 117.7, 127.9, 130.7, 142.7, 158.3, 160.4, 171.3. IR (film): v 2990, 2960, 2940, 2880, 1680, 1600, $1580 \mathrm{~cm}^{-1}$. Anal. Calcd. for $\mathrm{C}_{13} \mathrm{H}_{15} \mathrm{NO}_{3}$ : C. 66.94; H. 6.48; N. 6.00. Found: C. 66.76; H. 6.23; N. 5.87. 
Preparation of ethyl 3-(2,4-dimethoxybenzyl)-2-oxo-3-azabicyclo [3.1.0]hexane-6-carboxylate (10-trans and 10-cis) by tandem RCMcyclopropanation. A solution of catalyst II $(67.84 \mathrm{mg}, 0.08 \mathrm{mmol})$ in $5 \mathrm{~mL}$ of dry toluene was added via cannula to a refluxing solution of amide $8(200.0 \mathrm{mg}$, $0.8 \mathrm{mmol})$ in dry toluene $(20 \mathrm{mM})$ under argon. A solution of ethyl diazoacetate (698.3 mg, $6.1 \mathrm{mmol}$ ) in $10 \mathrm{~mL}$ of dry toluene was then added slowly during $8 \mathrm{~h}$. The resulting dark solution was then filtered by Celite and the solvent was eliminated under reduced pressure. The resulting mixture was purified first by flash chromatography (Petroleum ether:AcOEt 2:1) and then by MPLC (Petroleum ether:AcOEt 6:1), affording $87.9 \mathrm{mg} \mathrm{(36 \% )} \mathrm{of} \mathrm{10-trans} \mathrm{and} 54.8 \mathrm{mg}$ $(22 \%)$ of 10 -cis as pale yellow oils.<smiles>CCOC(=O)[C@H]1C[C@H]2CN(Cc3ccc(OC)cc3OC)C(=O)[C@@H]12</smiles>

Ethyl $\left(1 R^{*}, 5 S^{*}, 6 R^{*}\right)-3-(2,4-d i m e t h o x y b e n z y l)-2-o x o-3-a z a b i c y c l o[3.1 .0]$ hexane-6-carboxylate (10-trans). ${ }^{1} \mathrm{H}-\mathrm{NMR}\left(\mathrm{CDCl}_{3}, 300 \mathrm{MHz}\right): \delta 1.23$ (t, $\mathrm{J}=7.3$ Eliminado: RMN $\mathrm{Hz}, 3 \mathrm{H}), 1.29-1.31(\mathrm{~m}, 1 \mathrm{H}), 2.02-2.09(\mathrm{~m}, 1 \mathrm{H}), 2.46(\mathrm{~d}, \mathrm{~J}=18.3 \mathrm{~Hz}, 1 \mathrm{H}), 2.86$ (dd, $J_{1}=18.3 \mathrm{~Hz}, J_{2}=7.3 \mathrm{~Hz}, 1 \mathrm{H}$ ), $3.44(\mathrm{~d}, \mathrm{~J}=7.3 \mathrm{~Hz}, 1 \mathrm{H}$ ), $3.80(\mathrm{~s}, 3 \mathrm{H}), 3.81$ (s, $3 \mathrm{H}), 4.09$ (q, J= $7.3 \mathrm{~Hz}, 2 \mathrm{H}), 4.40(\mathrm{~d}, \mathrm{~J}=14.0 \mathrm{~Hz}, 1 \mathrm{H}), 4.47$ (d, J=14.0 Hz, 1H), 6.43-6.45 (m, 2H), $7.18(\mathrm{~d}, \mathrm{~J}=9.1 \mathrm{~Hz}, 1 \mathrm{H}) .{ }^{13} \mathrm{C}-\mathrm{NMR}\left(\mathrm{CDCl}_{3}, 75 \mathrm{MHz}\right): \delta 14.2$, 16.8, 29.6, 34.6, 41.1, 44.6, 55.2, 55.4, 60.6, 98.4, 104.0, 116.7, 131.3, 158.6, 160.8, 170.6, 173.4. IR (film): v 2960, 2920, 2840, 1720, 1690, 1610, $1585 \mathrm{~cm}^{-1}$. n.O.e. $\left(\mathrm{H}_{1.29-1.31} \rightarrow \mathrm{H}_{2.02-2.09} 0 \%\right.$; $\mathrm{H}_{1.29-1.31} \rightarrow \mathrm{H}_{2.46}$ 6.5\%). Anal. Calcd. for $\mathrm{C}_{17} \mathrm{H}_{21} \mathrm{NO}_{5}$ : C. 63.94; H. 6.63; N. 4.39. Found: C. 63.76; H. 6.43; N. 4.63.

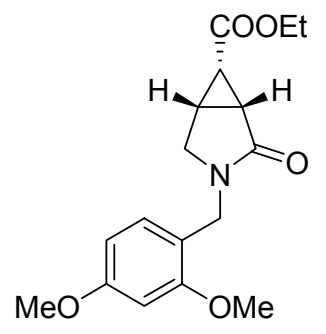


Ethyl $\left(1 R^{*}, 5 S^{*}, 6 S^{*}\right)-3-(2,4-d i m e t h o x y b e n z y l)-2-o x o-3-a z a b i c y c l o[3.1 .0]$ hexane-6-carboxylate (10-cis). ${ }^{1} \mathrm{H}-\mathrm{NMR}\left(\mathrm{CDCl}_{3}, 300 \mathrm{MHz}\right): \delta 1.26(\mathrm{t}, \mathrm{J}=7.3$ $\mathrm{Hz}, 3 \mathrm{H}), 1.23-1.27(\mathrm{~m}, 1 \mathrm{H}), 2.29-2.32(\mathrm{~m}, 1 \mathrm{H}), 2.45-2.47(\mathrm{~m}, 1 \mathrm{H}), 3.25\left(\mathrm{~d}, \mathrm{~J}_{1}=\right.$ $11.6 \mathrm{~Hz}, 1 \mathrm{H}), 3.48\left(\mathrm{dd}, \mathrm{J}_{1}=11.6 \mathrm{~Hz}, \mathrm{~J}_{2}=5.5 \mathrm{~Hz}, 1 \mathrm{H}\right), 3.80(\mathrm{~s}, 3 \mathrm{H}), 3.80(\mathrm{~s}, 3 \mathrm{H})$, $4.14(\mathrm{q}, \mathrm{J}=7.3 \mathrm{~Hz}, 2 \mathrm{H}), 4.20$ (d, J=14.0 Hz, 1H), 4.42 (d, J=14.0 Hz, 1H), 6.42$6.46(\mathrm{~m}, 2 \mathrm{H}), 7.08(\mathrm{~d}, \mathrm{~J}=8.6 \mathrm{~Hz}, 1 \mathrm{H}) .{ }^{13} \mathrm{C}-\mathrm{NMR}\left(\mathrm{CDCl}_{3}, 75 \mathrm{MHz}\right): \delta$ 14.2, 20.7, 26.0, 29.3, 40.4, 48.3, 55.3, 55.4, 61.1, 98.4, 104.2, 116.7, 131.0, 158.4, 160.6, 170.9, 171.5. IR (film): v 2960, 2920, 2840, 1720, 1690, 1610, $1585 \mathrm{~cm}^{-1}$. n.O.e. $\left(\mathrm{H}_{1.23-1.27} \rightarrow \mathrm{H}_{3.48} 5 \% ; \mathrm{H}_{1.23-1.27} \rightarrow \mathrm{H}_{2.45-2.47} \%\right)$ Anal. Calcd. for $\mathrm{C}_{17} \mathrm{H}_{21} \mathrm{NO}_{5}$ : C. 63.94; H. 6.63; N. 4.39. Found: C. 64.07; H. 6.78; N. 4.12.

Tandem RCM-isomerization in six members lactams. A solution of catalyst II (35.2 mg, $0.04 \mathrm{mmol})$ in $5 \mathrm{~mL}$ of dry toluene was added via cannula to a solution of 1 (114 $\mathrm{mg}, 0.4 \mathrm{mmol})$ in dry toluene $(20 \mathrm{mM})$ under argon. After $12 \mathrm{~h}$ the dark oil solution was filtered by Celite and the solvent was eliminated under reduced pressure. The resulting mixture was purified by flash chromatography (Hex:AcOEt 1:1), affording $64.8 \mathrm{mg} \mathrm{(64 \% )} \mathrm{of} \mathrm{4,} 17.2 \mathrm{mg}(17 \%)$ of 3 and $10.1 \mathrm{mg}(10 \%)$ of $\mathbf{2}$ as dark yellow oils.<smiles>COc1ccc(CN2CC=CCC2=O)c(OC)c1</smiles>

1-(2,4-dimethoxybenzyl)-3,6-dihydropyridin-2(1H)-one (2). ${ }^{1} \mathrm{H}-\mathrm{NMR}$ $\left(\mathrm{CDCl}_{3}, 300 \mathrm{MHz}\right): \delta 3.00-3.02(\mathrm{~m}, 2 \mathrm{H}), 3.80(\mathrm{~s}, 3 \mathrm{H}), 3.81(\mathrm{~s}, 3 \mathrm{H}), 3.83-3.87(\mathrm{~m}$, $2 \mathrm{H}), 4.63(\mathrm{~s}, 2 \mathrm{H}), 5.66-5.77(\mathrm{~m}, 2 \mathrm{H}), 6.44-6.46(\mathrm{~m}, 2 \mathrm{H}), 7.20(\mathrm{~d}, \mathrm{~J}=8.8 \mathrm{~Hz}, 1 \mathrm{H})$. ${ }^{13} \mathrm{C}-\mathrm{NMR}\left(\mathrm{CDCl}_{3}, 75 \mathrm{MHz}\right): \delta$ 32.2, 43.5, 48.4, 55.3 (2C), 98.3, 104.2, 117.2, 121.1, 122.3, 130.4, 158.6, 160.2, 167.2. IR (film): v 3040, 2980, 2960, 2920, 2820, 1630, 1610, $1580 \mathrm{~cm}^{-1}$. Anal. Calcd. for $\mathrm{C}_{14} \mathrm{H}_{17} \mathrm{NO}_{3}$ : C. 68.00; H. 6.93; N. 5.66. Found: C. 68.27 ; H. 6.76; N. 5.57.<smiles>COc1ccc(CN2CCC=CC2=O)c(OC)c1</smiles>

Eliminado: RMN

Eliminado: RMN

Con formato: Portugués

(Brasil)

Con formato: Portugués (Brasil)

Con formato: Portugués (Brasil)

Con formato: Portugués

(Brasil)

Eliminado: RMN

Con formato: Portugués (Brasil)

Eliminado: RMN 
1-(2,4-dimethoxybenzyl)-5,6-dihydropyridin-2(1H)-one (3). ${ }^{1} \mathrm{H}-\mathrm{NMR}$ $\left(\mathrm{CDCl}_{3}, 300 \mathrm{MHz}\right): \delta 2.28-2.34(\mathrm{~m}, 2 \mathrm{H}), 3.34-3.39(\mathrm{~m}, 2 \mathrm{H}), 3.80(\mathrm{~s}, 3 \mathrm{H}), 3.81(\mathrm{~s}$, $3 \mathrm{H}), 4.58(\mathrm{~s}, 2 \mathrm{H}), 5.96(\mathrm{~d}, \mathrm{~J}=9.9 \mathrm{~Hz}, 1 \mathrm{H}), 6.40(\mathrm{~m}, 2 \mathrm{H}), 6.50-6.56(\mathrm{~m}, 1 \mathrm{H}), 7.21$ $(\mathrm{d}, \mathrm{J}=8.8 \mathrm{~Hz}, 1 \mathrm{H}) \cdot{ }^{13} \mathrm{C}-\mathrm{NMR}\left(\mathrm{CDCl}_{3}, 75 \mathrm{MHz}\right): \delta 24.2,43.9,44.8,55.3,55.3$, 98.3, 104.2, 117.9, 125.6, 130.4, 139.1, 158.6, 160.1, 164.6. IR (film): v 2920, 2820, 1660, 1610, 1580, $1510 \mathrm{~cm}^{-1}$. Anal. Calcd. for $\mathrm{C}_{14} \mathrm{H}_{17} \mathrm{NO}_{3}$ : C. $68.00 ; \mathrm{H}$. 6.93; N. 5.66. Found: C. 68.29; H. 6.82; N. 5.54.<smiles>COc1ccc(CN2C=CCCC2=O)c(OC)c1</smiles>

\section{1-(2,4-dimethoxybenzyl)-3,4-dihydropyridin-2(1H)-one}

(4). ${ }^{1} \mathrm{H}-\mathrm{NMR}$ $\left(\mathrm{CDCl}_{3}, 300 \mathrm{MHz}\right): \delta 2.27-2.33(\mathrm{~m}, 2 \mathrm{H}), 2.52-2.57(\mathrm{~m}, 2 \mathrm{H}), 3.79(\mathrm{~s}, 3 \mathrm{H}), 3.80(\mathrm{~s}$, $3 \mathrm{H}), 4.62(\mathrm{~s}, 2 \mathrm{H}), 5.05-5.10(\mathrm{~m}, 1 \mathrm{H}), 6.08-6.12(\mathrm{~m}, 1 \mathrm{H}), 6.42-6.46(\mathrm{~m}, 2 \mathrm{H}), 7.15$ $(\mathrm{d}, \mathrm{J}=8.8 \mathrm{~Hz}, 1 \mathrm{H}) .{ }^{13} \mathrm{C}-\mathrm{NMR}\left(\mathrm{CDCl}_{3}, 75 \mathrm{MHz}\right): \delta 20.3,31.4,43.4,55.2,55.3$, 98.3, 103.9, 105.5, 117.7, 130.0, 130.3, 158.3, 160.2, 169.3. IR (film): v 2980, 2940, 2910, 2820, 1660, 1610, 1580, $1500 \mathrm{~cm}^{-1}$. Anal. Calcd. for $\mathrm{C}_{14} \mathrm{H}_{17} \mathrm{NO}_{3}$ : C. 68.00; H. 6.93; N. 5.66. Found: C. 68.11; H. 7.12; N. 5.54.

General procedure for the synthesis of cyclopropanes from isolated unsaturated lactams using ethyl diazoacetate. A solution of catalyst II (33.9 $\mathrm{mg}, 0.04 \mathrm{mmol}$ ) in $5 \mathrm{~mL}$ of dry toluene was added via cannula to a refluxing solution of the unsaturated lactam $(0.4 \mathrm{mmol})$ in dry toluene $(20 \mathrm{mM})$ under argon. A solution of ethyl diazoacetate $(369.5 \mathrm{mg}, 3.24 \mathrm{mmol})$ in $10 \mathrm{~mL}$ of dry toluene was then added slowly during $8 \mathrm{~h}$. The resulting dark solution was then filtered by Celite and the solvent was eliminated under reduced pressure. The resulting mixture was purified first by flash chromatography and then by MPLC.

Preparation of ethyl 2-(2,4-dimethoxybenzyl)-3-oxo-2-azabicyclo [4.1.0]heptane-7-carboxylate (6-trans and 6-cis). Following the typical procedure for the synthesis of cyclopropanes from isolated unsaturated lactams using ethyl diazoacetate, the reaction of $4(100.0 \mathrm{mg}, 0.4 \mathrm{mmol})$ afforded after flash chromatography (Petroleum ether:AcOEt 2:1) followed of MPLC
Eliminado: RMN

Eliminado: RMN 
(Petroleum ether:AcOEt 6:1) $47.4 \mathrm{mg}$ (36\%) of 6-trans and $32.6 \mathrm{mg} \mathrm{(25 \% )} \mathrm{of} \mathrm{6-}$ cis as pale yellow oils, and $23 \mathrm{mg}(17 \%)$ of 7 as a yellow oil.

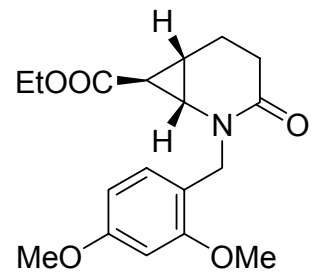

Ethyl $\left(1 S^{*}, 6 S^{*}, 7 S^{*}\right)-2-(2,4-d i m e t h o x y b e n z y l)-3-0 x o-2-a z a b i c y c l o[4.1 .0]$ heptane-7-carboxylate (6-trans). ${ }^{1} \mathrm{H}-\mathrm{NMR}\left(\mathrm{CDCl}_{3}, 300 \mathrm{MHz}\right): \delta 1.22(\mathrm{t}, \mathrm{J}=7.1$ Eliminado: RMN $\mathrm{Hz}, 3 \mathrm{H}) ; 1.43-1.45(\mathrm{~m}, 1 \mathrm{H}), 1.61-1.69(\mathrm{~m}, 1 \mathrm{H}), 1.88-1.92(\mathrm{~m}, 1 \mathrm{H}), 2.17-2.22(\mathrm{~m}$, $1 \mathrm{H}), 2.28-2.46(\mathrm{~m}, 2 \mathrm{H}), 3.05\left(\mathrm{dd}, \mathrm{J}_{1}=8.8 \mathrm{~Hz}, J_{2}=2.2 \mathrm{~Hz}, 1 \mathrm{H}\right), 3.75(\mathrm{~s}, 3 \mathrm{H})$, $3.79(\mathrm{~s}, 3 \mathrm{H}), 4.02-4.07(\mathrm{~m}, 2 \mathrm{H}), 4.57(\mathrm{~d}, \mathrm{~J}=14.3 \mathrm{~Hz}, 1 \mathrm{H}), 4.65(\mathrm{~d}, \mathrm{~J}=14.3 \mathrm{~Hz}$, $1 \mathrm{H}), 6.42-6.45(\mathrm{~m}, 2 \mathrm{H}), 7.19(\mathrm{~d}, \mathrm{~J}=8.8 \mathrm{~Hz}, 1 \mathrm{H}) .{ }^{13} \mathrm{C}-\mathrm{NMR}\left(\mathrm{CDCl}_{3}, 75 \mathrm{MHz}\right): \delta$ $14.1,21.0,21.7,30.7,30.9,40.8,43.3,55.1,55.3,60.5,98.2,104.0,117.1$, 131.5, 158.7, 160.5, 170.5, 171.2. IR (film): $v$ 3065, 2985, 1600, 1565, $1440 \mathrm{~cm}^{-}$ 1.n.O.e. $\left(\mathrm{H}_{1.61-1.69} \rightarrow \mathrm{H}_{1.43-1.45} 0 \% ; \mathrm{H}_{1.61-1.69} \rightarrow \mathrm{H}_{3.05}\right.$ 9\%) Anal. Calcd. for $\mathrm{C}_{18} \mathrm{H}_{23} \mathrm{NO}_{5}$ : C. 64.85 ; H. 6.95; N. 4.20. Found: C. 64.96; H. 7.17; N. 3.98.

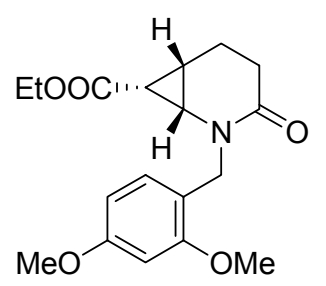

Ethyl $\left(1 S^{*}, 6 S^{*}, 7 R^{*}\right)-2-(2,4-d i m e t h o x y b e n z y l)-3-o x o-2-a z a b i c y c l o[4.1 .0]$ heptane-7-carboxylate (6-cis). ${ }^{1} \mathrm{H}-\mathrm{NMR}\left(\mathrm{CDCl}_{3}, 300 \mathrm{MHz}\right): \delta 1.25$ (t, $\mathrm{J}=7.1$ $\mathrm{Hz}, 3 \mathrm{H}), 1.67\left(\mathrm{dd}, \mathrm{J}_{1}=8.8, \mathrm{~J}_{2}=6.6 \mathrm{~Hz}, 1 \mathrm{H}\right), 1.82-1.86(\mathrm{~m}, 1 \mathrm{H}), 2.09-2.17(\mathrm{~m}$, $1 \mathrm{H}), 2.28-2.42(\mathrm{~m}, 3 \mathrm{H}), 3.09\left(\mathrm{dd}, J_{1}=8.3, J_{2}=7.1 \mathrm{~Hz}, 1 \mathrm{H}\right), 3.79(\mathrm{~s}, 3 \mathrm{H}), 3.80(\mathrm{~s}$, $3 \mathrm{H}), 3.90(\mathrm{~d}, \mathrm{~J}=14.3 \mathrm{~Hz}, 1 \mathrm{H}), 4.03-4.15(\mathrm{~m}, 2 \mathrm{H}), 4.99(\mathrm{~d}, \mathrm{~J}=14.3 \mathrm{~Hz}, 1 \mathrm{H}), 6.42-$ $6.45(\mathrm{~m}, 2 \mathrm{H}), 7.22(\mathrm{~d}, \mathrm{~J}=9.3 \mathrm{~Hz}, 1 \mathrm{H}) .{ }^{13} \mathrm{C}-\mathrm{NMR}\left(\mathrm{CDCl}_{3}, 75 \mathrm{MHz}\right): \delta 14.2,18.2$, 24.3, 29.7, 31.7, 38.7, 43.2, 55.3, 55.3, 60.3, 98.2, 104.0, 117.0, 131.7, 158.8, 160.3, 169.2, 171.7. IR (film): v 3085, 2985, 1600, 1565, $1440 \mathrm{~cm}^{-1}$. n.O.e. $\left(\mathrm{H}_{3.09} \rightarrow \mathrm{H}_{1.67} 7 \% ; \mathrm{H}_{1.82-1.86} \rightarrow \mathrm{H}_{1.67} 5 \%\right)$. Anal. Calcd. for $\mathrm{C}_{18} \mathrm{H}_{23} \mathrm{NO}_{5}$ : C. 64.85; H. 6.95; N. 4.20. Found: C. 64.77 ; H. 6.74; N. 3.99. 
<smiles>CCOC(=O)CC1=CN(Cc2ccc(OC)cc2OC)C(=O)CC1</smiles>

Ethyl [1-(2,4-dimethoxybenzyl)-6-oxo-1,4,5,6-tetrahydropyridin-3yl]acetate (7). ${ }^{1} \mathrm{H}-\mathrm{NMR}\left(\mathrm{CDCl}_{3}, 300 \mathrm{MHz}\right): \delta 1.25(\mathrm{t}, \mathrm{J}=7.1 \mathrm{~Hz}, 3 \mathrm{H}), 2.35(\mathrm{t}, \mathrm{J}=$ $7.7 \mathrm{~Hz}, 2 \mathrm{H}), 2.58(\mathrm{t}, \mathrm{J}=7.7 \mathrm{~Hz}, 2 \mathrm{H}), 2.99(\mathrm{~s}, 2 \mathrm{H}), 3.80(\mathrm{~s}, 3 \mathrm{H}), 3.81(\mathrm{~s}, 3 \mathrm{H})$, 4.10-4.16 (m, 2H), 4.61 (s, 2H), $6.02(\mathrm{~s}, 1 \mathrm{H}), 6.42-6.45(\mathrm{~m}, 2 \mathrm{H}), 7.15$ (d, J= 8.8 $\mathrm{Hz}, 1 \mathrm{H}$ ). IR (film): v 2980, 2940, 2910, 2830, 2815, 1730, 1660, 1610, 1580, $1510 \mathrm{~cm}^{-1}$. Anal. Calcd. for $\mathrm{C}_{18} \mathrm{H}_{23} \mathrm{NO}_{5}$ : C. 64.85 ; H. 6.95; N. 4.20. Found: C. 65.03; H. 6.81; N. 4.32.

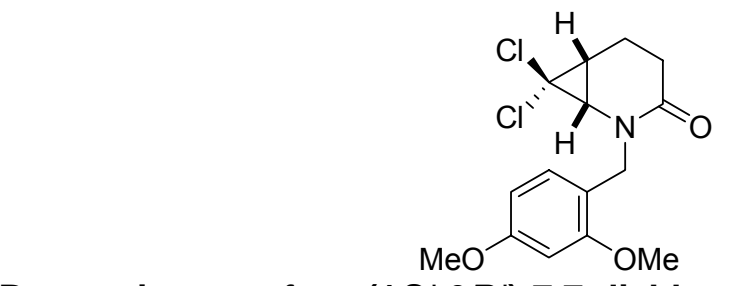

Preparation of $\left(1 S^{*}, 6 R^{*}\right)-7,7-d i c h l o r o-2-(2,4-d i m e t h o x y b e n z y l)-2-$ azabicyclo[4.1.0]heptan-3-one (5). A solution of catalyst II (30.9 mg, 0.04 $\mathrm{mmol}$ ) in $5 \mathrm{~mL}$ of dry toluene was added via cannula to a refluxing solution of amide 1 (150.0 mg, $0.6 \mathrm{mmol}$ ) in dry toluene $(20 \mathrm{mM})$ under argon. After $8 \mathrm{~h}$ the resulting dark brown solution was cooled to room temperature and then aliquat 336 (30 mg, $0.07 \mathrm{mmol}), \mathrm{DCM}(30 \mathrm{~mL}$ ) and $500 \mathrm{mg} \mathrm{NaOH}$ (50\% in water) were added. The reaction was sonicated for $12 \mathrm{~h}$. $15 \mathrm{~mL}$ of $\mathrm{NH}_{4} \mathrm{Cl}$ saturated were added, the crude was extracted with $\mathrm{DCM}(3 \times 10 \mathrm{~mL})$, dried with $\mathrm{MgSO}_{4}$, filtered by Celite and the solvent was eliminated under reduced pressure. The resulting mixture was purified by flash chromatography (Hex:AcOEt 2:1), affording $65.2 \mathrm{mg}$ (55\%) of 5 as a yellow oil.

${ }^{1} \mathrm{H}-\mathrm{NMR}\left(\mathrm{CDCl}_{3}, 300 \mathrm{MHz}\right): \delta 1.89-2.01(\mathrm{~m}, 1 \mathrm{H}), 2.06-2.15(\mathrm{~m}, 1 \mathrm{H}), 2.19-$ $2.44(\mathrm{~m}, 3 \mathrm{H}), 3.10(\mathrm{~d}, \mathrm{~J}=9.9 \mathrm{~Hz}, 1 \mathrm{H}), 3.82(\mathrm{~s}, 3 \mathrm{H}), 3.85(\mathrm{~s}, 3 \mathrm{H}), 4.19$ (d, J= 14.3 $\mathrm{Hz}, 1 \mathrm{H}), 5.14(\mathrm{~d}, \mathrm{~J}=14.3 \mathrm{~Hz}, 1 \mathrm{H}), 6.48$ (bs, $2 \mathrm{H}), 7.30$ (d, J=8.8 Hz, $1 \mathrm{H}) .{ }^{13} \mathrm{C}-$ 
$\mathrm{NMR}\left(\mathrm{CDCl}_{3}, 75 \mathrm{MHz}\right): \delta 19.5,28.3,30.5,42.7,44.0,55.3(2 \mathrm{C}), 65.0,98.1$, 104.1, 116.6, 132.2, 158.9, 160.6, 170.4. IR (film): v 3000, 2915, 2815, 1670 , 1610, $1580 \mathrm{~cm}^{-1}$. Anal. Calcd. for $\mathrm{C}_{15} \mathrm{H}_{17} \mathrm{Cl}_{2} \mathrm{NO}_{3}$ : C. 54.56; H. 5.19; N. 4.24. Found: C. 54.69; H. 5.32; N. 4.16.

Tandem RCM-isomerization in seven members lactams. A solution of catalyst II $(87.7 \mathrm{mg}, 0.1 \mathrm{mmol})$ in $5 \mathrm{~mL}$ of dry toluene was added via cannula to a solution of $11(300.0 \mathrm{mg}, 1.0 \mathrm{mmol})$ in dry toluene $(20 \mathrm{mM})$ under argon . After $24 \mathrm{~h}$ the dark oil solution was filtered by Celite and the solvent was eliminated under reduced pressure. The resulting mixture was purified by flash chromatography (Hex:AcOEt 1:1), affording $167.0 \mathrm{mg}$ (62\%) of 12 as a pale yellow oil, $54.8 \mathrm{mg} \mathrm{(20 \% )}$ of 13 as a dark yellow oil, $14.6 \mathrm{mg} \mathrm{(5 \% )} \mathrm{of} 14$ and $12.0 \mathrm{mg} \mathrm{(4 \% )}$ of 15 as yellow oils.

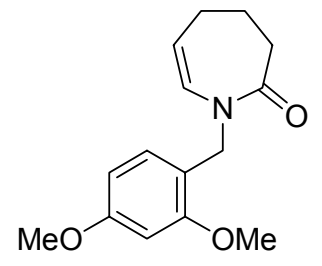

1-(2,4-dimethoxybenzyl)-1,3,4,5-tetrahydro-2H-azepin-2-one (12). ${ }^{1} \mathrm{H}$ NMR $\left(\mathrm{CDCl}_{3}, 300 \mathrm{MHz}\right): \delta$ 1.99-2.08 (m, 2H), 2.14-2.20 (m, 2H), 2.56-2.60 (m, $2 \mathrm{H}), 3.78(\mathrm{~s}, 3 \mathrm{H}), 3.79(\mathrm{~s}, 3 \mathrm{H}), 4.61(\mathrm{~s}, 2 \mathrm{H}), 5.23-5.30(\mathrm{~m}, 1 \mathrm{H}), 5.91-5.95(\mathrm{~m}$, $1 \mathrm{H}), 6.42(\mathrm{~m}, 2 \mathrm{H}), 7.15(\mathrm{~d}, \mathrm{~J}=8.8 \mathrm{~Hz}, 1 \mathrm{H}) .{ }^{13} \mathrm{C}-\mathrm{NMR}\left(\mathrm{CDCl}_{3}, 75 \mathrm{MHz}\right) ; \delta 26.1$, 26.3, 36.0, 44.7, 55.2, 55.2, 98.2, 103.9, 116.6, 117.9, 130.0, 130.0, 158.2, 160.0, 174.1. IR (film): v 2990, 2920, 2820, 1650, 1610, $1580 \mathrm{~cm}^{-1}$. Anal. Calcd. for $\mathrm{C}_{15} \mathrm{H}_{19} \mathrm{NO}_{3}$ : C. 68.94; H. 7.33; N. 5.36. Found: C. 69.06; H. 7.48; N. 5.23.

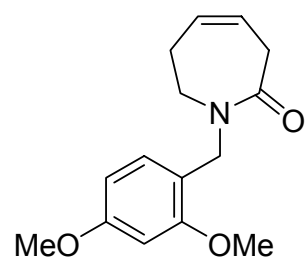

1-(2,4-dimethoxybenzyl)-1,3,6,7-tetrahydro-2H-azepin-2-one (13). ${ }^{1} \mathrm{H}$ NMR $\left(\mathrm{CDCl}_{3}, 300 \mathrm{MHz}\right): \delta$ 2.11-2.14 (m, 2H), 3.26-3.29 (m, 2H), 3.52-3.56 (m, 
$2 \mathrm{H}), 3.79(\mathrm{~s}, 3 \mathrm{H}), 3.80(\mathrm{~s}, 3 \mathrm{H}), 4.57$ (s, 2H), 5.55-5.64 (bs, 2H), 6.41-6.45 (m, $2 \mathrm{H}), 7.18(\mathrm{~d}, \mathrm{~J}=8.8 \mathrm{~Hz}, 1 \mathrm{H}) .{ }^{13} \mathrm{C}-\mathrm{NMR}\left(\mathrm{CDCl}_{3}, 75 \mathrm{MHz}\right): \delta 28.5,35.9,43.8$, 45.4, 55.2, 55.3, 98.1, 104.1, 118.2, 120.9, 129.0, 130.5, 158.3, 160.1, 173.6. IR (film): v 3060, 3010, 2990, 2920, 2820, 1640, 1610, $1580 \mathrm{~cm}^{-1}$. Anal. Calcd. for $\mathrm{C}_{15} \mathrm{H}_{19} \mathrm{NO}_{3}$ : C. 68.94; H. 7.33; N. 5.36. Found: C. 68.79; H. 7.42; N. 5.15.

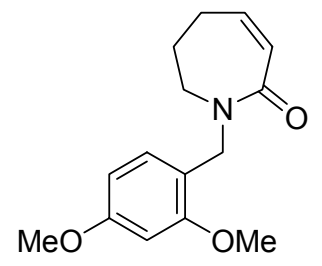

1-(2,4-dimethoxybenzyl)-1,5,6,7-tetrahydro-2H-azepin-2-one (14). ${ }^{1} \mathrm{H}$ NMR $\left(\mathrm{CDCl}_{3}, 300 \mathrm{MHz}\right): \delta$ 1.73-1.81 (m, 2H), 2.22-2.29 (m, 2H), 3.33-3.36 (m, $2 \mathrm{H}), 3.80(\mathrm{~s}, 3 \mathrm{H}), 3.81(\mathrm{~s}, 3 \mathrm{H}), 4.62(\mathrm{~s}, 2 \mathrm{H}), 6.03(\mathrm{~d}, \mathrm{~J}=12.1 \mathrm{~Hz}, 1 \mathrm{H}), 6.16-6.23$ $(\mathrm{m}, 1 \mathrm{H}), 6.42-6.46(\mathrm{~m}, 2 \mathrm{H}), 7.23(\mathrm{~d}, \mathrm{~J}=8.8 \mathrm{~Hz}, 1 \mathrm{H}) .{ }^{13} \mathrm{C}-\mathrm{NMR}\left(\mathrm{CDCl}_{3}, 75 \mathrm{MHz}\right):$ $\delta$ 27.8, 28.3, 45.2, 46.7, 55.2, 55.3, 98.2, 104.0, 118.1, 124.7, 130.9, 137.8, 158.4, 160.1, 168.8. IR (film): v 2920, 2820, 1630, 1610, $1580 \mathrm{~cm}^{-1}$. Anal. Calcd. for $\mathrm{C}_{15} \mathrm{H}_{19} \mathrm{NO}_{3}$ : C. 68.94 ; H. 7.33; N. 5.36. Found: C. 69.06; H. 7.48; N. 5.23 .

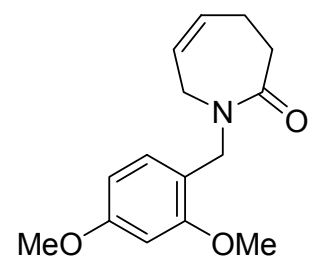

1-(2,4-dimethoxybenzyl)-1,3,4,7-tetrahydro-2H-azepin-2-one (15). ${ }^{1} \mathrm{H}$ NMR $\left(\mathrm{CDCl}_{3}, 300 \mathrm{MHz}\right): \delta 2.39-2.45(\mathrm{~m}, 2 \mathrm{H}), 2.76-2.80(\mathrm{~m}, 2 \mathrm{H}), 3.80(\mathrm{~s}, 6 \mathrm{H})$, 3.83-3.86 (m, 2H), $4.57(\mathrm{~s}, 2 \mathrm{H}), 5.58-5.74(\mathrm{~m}, 2 \mathrm{H}), 6.42-6.46(\mathrm{~m}, 2 \mathrm{H}), 7.15(\mathrm{~d}$, $\mathrm{J}=8.8 \mathrm{~Hz}, 1 \mathrm{H}) .{ }^{13} \mathrm{C}-\mathrm{NMR}\left(\mathrm{CDCl}_{3}, 75 \mathrm{MHz}\right): \delta 25.2,33.8,45.5,45.7,55.2,55.3$, 98.2, 103.9, 118.1, 124.7, 130.2, 131.1, 158.4, 160.1, 174.7. IR (film): v 3010, 2990, 2920, 2820, 1640, 1610, $1580 \mathrm{~cm}^{-1}$. Anal. Calcd. for $\mathrm{C}_{15} \mathrm{H}_{19} \mathrm{NO}_{3}$ : C. 68.94; H. 7.33; N. 5.36. Found: C. 69.01; H. 7.27; N. 5.41.

Preparation of ethyl 2-(2,4-dimethoxybenzyl)-3-oxo-2-azabicyclo [5.1.0]octane-8-carboxylate (16-cis and 16-trans). Following the typical
Eliminado: RMN
Eliminado: RMN

Eliminado: RMN 
procedure for the synthesis of cyclopropanes from isolated unsaturated lactams using ethyl diazoacetate, the reaction of $12(250.0 \mathrm{mg}, 1.0 \mathrm{mmol})$ afforded after flash chromatography (Petroleum ether:AcOEt 2:1) $200 \mathrm{mg}(60 \%)$ of 16-trans and 16-cis (4:1 mixture as calculated from well resolved signals in the H NMR spectrum), isolating only 16-trans after MPLC (Petroleum ether:AcOEt 4:1), We also detected the signals corresponding to $\mathbf{1 7}$ in the crude mixture.

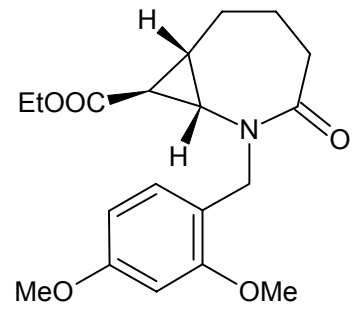

Ethyl $\left(1 S^{*}, 7 S^{*}, 8 S^{*}\right)-2-(2,4-d i m e t h o x y b e n z y l)-3-o x o-2-a z a b i c y c l o[5.1 .0]$ octane-8-carboxylate (16-trans). ${ }^{1} \mathrm{H}-\mathrm{NMR}\left(\mathrm{CDCl}_{3}, 300 \mathrm{MHz}\right): \delta 1.33 \mathrm{(t}, \mathrm{J}=$ 7.3Hz, 3H), 1.55-1.66 (m, 1H), 1.77-1.88 (m, 3H), 1.95-2.05 (m, 1H), 2.18-2.44 $(\mathrm{m}, 2 \mathrm{H}), 2.70-2.81(\mathrm{~m}, 1 \mathrm{H}), 2.94(\mathrm{t}, \mathrm{J}=6.7 \mathrm{~Hz}, 1 \mathrm{H}), 3.74(\mathrm{~d}, \mathrm{~J}=14.6 \mathrm{~Hz}, 1 \mathrm{H})$, $3.80(\mathrm{~s}, 6 \mathrm{H}), 4.15-4.24(\mathrm{~m}, 2 \mathrm{H}), 5.18(\mathrm{~d}, \mathrm{~J}=14.6 \mathrm{~Hz}, 1 \mathrm{H}), 6.43-6.45(\mathrm{~m}, 2 \mathrm{H})$, $7.18(\mathrm{~d}, \mathrm{~J}=8.6 \mathrm{~Hz}, 1 \mathrm{H}) .{ }^{13} \mathrm{C}-\mathrm{NMR}\left(\mathrm{DMSO}-\mathrm{d}_{6}, 75 \mathrm{MHz}\right.$ ); $\delta 14.2,17.0,20.5,22.7$, 23.4, 30.7, 33.7, 42.9, 55.2, 55.4, 60.0, 98.3, 104.5, 117.0, 129.8, 158.2, 159.8, 169.7, 172.5. IR (film): $\vee$ 3080, 2980, 1610, 1565, $1440 \mathrm{~cm}^{-1}$. n.O.e. $\left(\mathrm{H}_{2.94} \rightarrow\right.$ $\mathrm{H}_{1.55-1.66} 0 \% ; \mathrm{H}_{2.94} \rightarrow \mathrm{H}_{1.77-1.88} 6 \%$ ) Anal. Calcd. for $\mathrm{C}_{19} \mathrm{H}_{25} \mathrm{NO}_{5}$ : C. 65.69; $\mathrm{H}$. 7.25; N. 4.03. Found: C. 65.76; H. 7.06; N. 3.94.

General procedure for the synthesis of cyclopropanes by tandem RCM-isomerization-cyclopropanation. A solution of catalyst II $(101.8 \mathrm{mg}$, $0.12 \mathrm{mmol}$ ) in $5 \mathrm{~mL}$ of dry toluene was added via cannula to a solution of the corresponding $\mathrm{N}$-allylamide $(0.8 \mathrm{mmol})$ in dry toluene $(20 \mathrm{mM})$ under argon. A solution of ethyl diazoacetate $(698.3 \mathrm{mg}, 6.1 \mathrm{mmol})$ in $10 \mathrm{~mL}$ of dry toluene was then added slowly during $8 \mathrm{~h}$. The resulting dark solution was then filtered by Celite and the solvent was eliminated under reduced pressure. The resulting mixture was purified first by flash chromatography and then by MPLC.

Preparation of ethyl 2-(2,4-dimethoxybenzyl)-3-oxo-2-azabicyclo [4.1.0]heptane-7-carboxylate (6-trans and 6-cis). Following the general procedure for the synthesis of cyclopropanes by tandem RCM-isomerization- 
cyclopropanation, the reaction of $1(100.0 \mathrm{mg}, 0.4 \mathrm{mmol})$ afforded after flash chromatography (Petroleum ether:AcOEt 2:1) followed of MPLC (Petroleum ether:AcOEt 6:1) $45.8 \mathrm{mg}$ (34\%) of 6-trans and $24.1 \mathrm{mg} \mathrm{(18 \% )} \mathrm{of} \mathrm{6-cis} \mathrm{as} \mathrm{pale}$ yellow oils. 7 was detected by t.I.c. and NMR but was not isolated.

Preparation of ethyl 4-(2,4-dimethoxybenzyl)-3-oxo-4-azabicyclo [5.1.0]octane-8-carboxylate (18-cis and 18-trans) and ethyl 3-(2,4dimethoxybenzyl)-4-oxo-3-azabicyclo[5.1.0]octane-8-carboxylate (19-cis and 19-trans). Following the general procedure for the synthesis of cyclopropanes by tandem RCM-isomerization-cyclopropanation, the reaction of 11 (200.0 mg, $0.7 \mathrm{mmol}$ ) afforded after flash chromatography (Petroleum ether:AcOEt 2:1) $80 \mathrm{mg}$ (34\%) of 18-trans and 18-cis as a $4: 1$ mixture, and $44.3 \mathrm{mg}(18 \%)$ of 19-trans and 19-cis as a $2: 1$ mixture, both as yellow oils. MPLC (Petroleum ether:AcOEt 6:1) allowed isolating only 18-trans and 19trans.

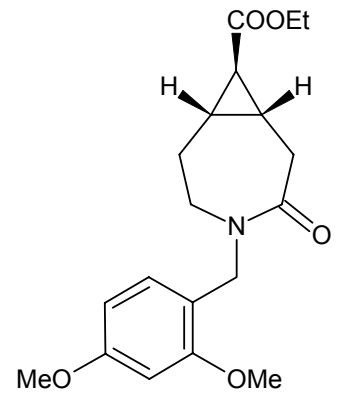

Ethyl $\left(1 R^{*}, 7 S^{*}, 8 R^{*}\right)-4-(2,4-d i m e t h o x y b e n z y l)-3-0 x o-4-a z a b i c y c l o[5.1 .0]$ octane-8-carboxylate (18-trans).

${ }^{1} \mathrm{H}-\mathrm{NMR}\left(\mathrm{CDCl}_{3}, 300 \mathrm{MHz}\right): \delta 1.26(\mathrm{t}, \mathrm{J}=7.0 \mathrm{~Hz}, 3 \mathrm{H}), 1.46(\mathrm{t}, \mathrm{J}=4.9 \mathrm{~Hz}$, Eliminado: RMN $1 \mathrm{H}), 1.54-1.61(\mathrm{~m}, 1 \mathrm{H}), 1.71-1.82(\mathrm{~m}, 2 \mathrm{H}), 1.86-1.94(\mathrm{~m}, 1 \mathrm{H}), 2.71\left(\mathrm{dd}, J_{1}=14.6\right.$ $\left.\mathrm{Hz}, \mathrm{J}_{2}=6.7 \mathrm{~Hz}, 1 \mathrm{H}\right), 3.04-3.17(\mathrm{~m}, 2 \mathrm{H}), 3.31-3.40(\mathrm{~m}, 1 \mathrm{H}), 3.81(\mathrm{~s}, 3 \mathrm{H}), 3.82(\mathrm{~s}$, $3 \mathrm{H}), 4.10-4.15(\mathrm{~m}, 2 \mathrm{H}), 4.33(\mathrm{~d}, \mathrm{~J}=14.6 \mathrm{~Hz}, 1 \mathrm{H}), 4.68(\mathrm{~d}, \mathrm{~J}=14.6 \mathrm{~Hz}, 1 \mathrm{H}), 6.45$ $(\mathrm{m}, 2 \mathrm{H}), 7.16(\mathrm{~d}, \mathrm{~J}=9.1 \mathrm{~Hz}, 1 \mathrm{H}){ }^{13} \mathrm{C}-\mathrm{NMR}\left(\mathrm{CDCl}_{3}, 75 \mathrm{MHz}\right): \delta$ 14.2, 20.7, 22.4, 26.2, 29.7, 35.1, 44.0, 44.8, 55.3, 55.3, 60.6, 98.3, 104.0, 117.7, 131.0, 158.5, 160.4, 171.0, 173.9 IR (film): v 2940, 2900, 2820, 1720, 1640, 1610, 1580, 
1500, $1450 \mathrm{~cm}^{-1}$.n.O.e. $\left(\mathrm{H}_{1.46} \rightarrow \mathrm{H}_{1.71-1.82} 0 \%\right)$. Anal. Calcd. for $\mathrm{C}_{19} \mathrm{H}_{25} \mathrm{NO}_{5}$ : C. 65.69; H. 7.25; N. 4.03. Found: C. 65.88; H. 7.03; N. 4.24.

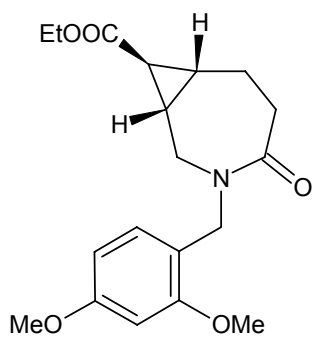

Ethyl (1S*,7R*,8S*)-3-(2,4-dimethoxybenzyl)-4-oxo-3-azabicyclo [5.1.0] octane-8-carboxylate (19-trans)

${ }^{1} \mathrm{H}-\mathrm{NMR}\left(\mathrm{CDCl}_{3}, 300 \mathrm{MHz}\right): \delta 1.26(\mathrm{t}, \mathrm{J}=7.1 \mathrm{~Hz}, 3 \mathrm{H}), 1.48(\mathrm{t}, \mathrm{J}=4.3 \mathrm{~Hz}$, $1 \mathrm{H}), 1.61-1.67(\mathrm{~m}, 1 \mathrm{H}), 2.00-2.07(\mathrm{~m}, 2 \mathrm{H}), 2.17-2.48(\mathrm{~m}, 3 \mathrm{H}), 3.36\left(\mathrm{dd}, \mathrm{J}_{1}=15.9\right.$ $\left.\mathrm{Hz}, J_{2}=7.3 \mathrm{~Hz}, 1 \mathrm{H}\right), 3.77-3.85(\mathrm{~m}, 1 \mathrm{H}), 3.81(\mathrm{~s}, 3 \mathrm{H}), 3.82(\mathrm{~s}, 3 \mathrm{H}), 4.10(\mathrm{q}, \mathrm{J}=$ $7.3 \mathrm{~Hz}, 2 \mathrm{H}), 4.41$ (d, J= $14.0 \mathrm{~Hz}, 1 \mathrm{H}), 4.68$ (d, J= $14.0 \mathrm{~Hz}, 1 \mathrm{H}), 6.45$ (bs, 2H), $7.20(\mathrm{~d}, \mathrm{~J}=9.1 \mathrm{~Hz}, 1 \mathrm{H}){ }^{13} \mathrm{C}-\mathrm{NMR}\left(\mathrm{CDCl}_{3}, 75 \mathrm{MHz}\right): \delta$ 14.2, 20.8, 22.7, 24.7, Eliminado: RMN 25.8, 32.7, 45.2, 46.9, 55.3, 55.3, 60.6, 98.4, 104.2, 117.9, 131.2, 158.5, 160.4, 173.4, 173.8 IR (film): y 2940, 2900, 2840, 1720, 1640, 1610, 1500, $1450 \mathrm{~cm}^{-}$ 1.n.O.e. $\left(\mathrm{H}_{1.48} \rightarrow \mathrm{H}_{1.61-1.67}\right.$ 0\%). Anal. Calcd. for $\mathrm{C}_{19} \mathrm{H}_{25} \mathrm{NO}_{5}$ : C. 65.69; H. 7.25; N. 4.03. Found: C. 65.92 ; H. 6.89; N. 3.85. 
1-(2,4-dimethoxybenzyl)-1,5-dihydro-2H-pyrrol-2-one (9):

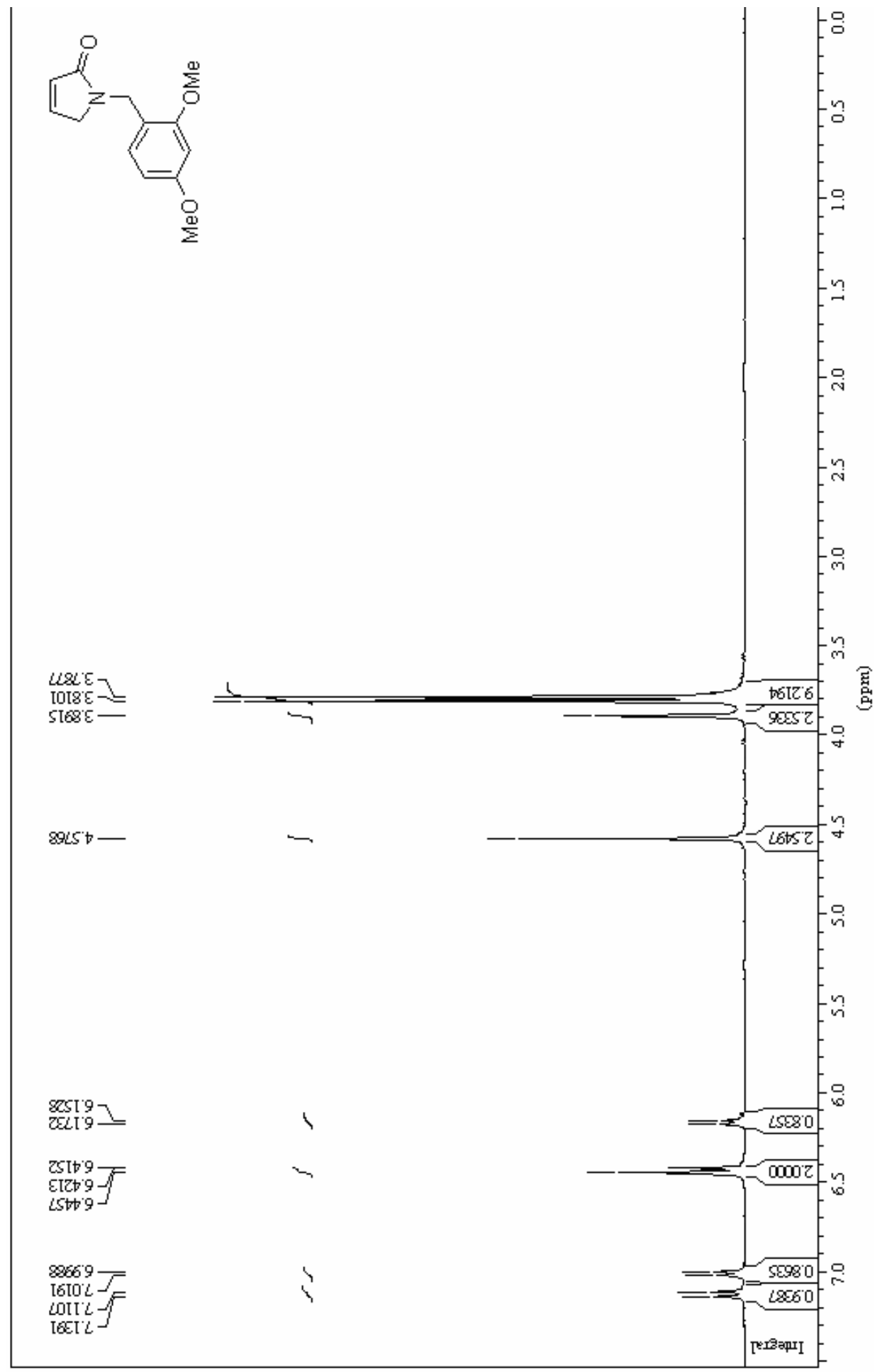




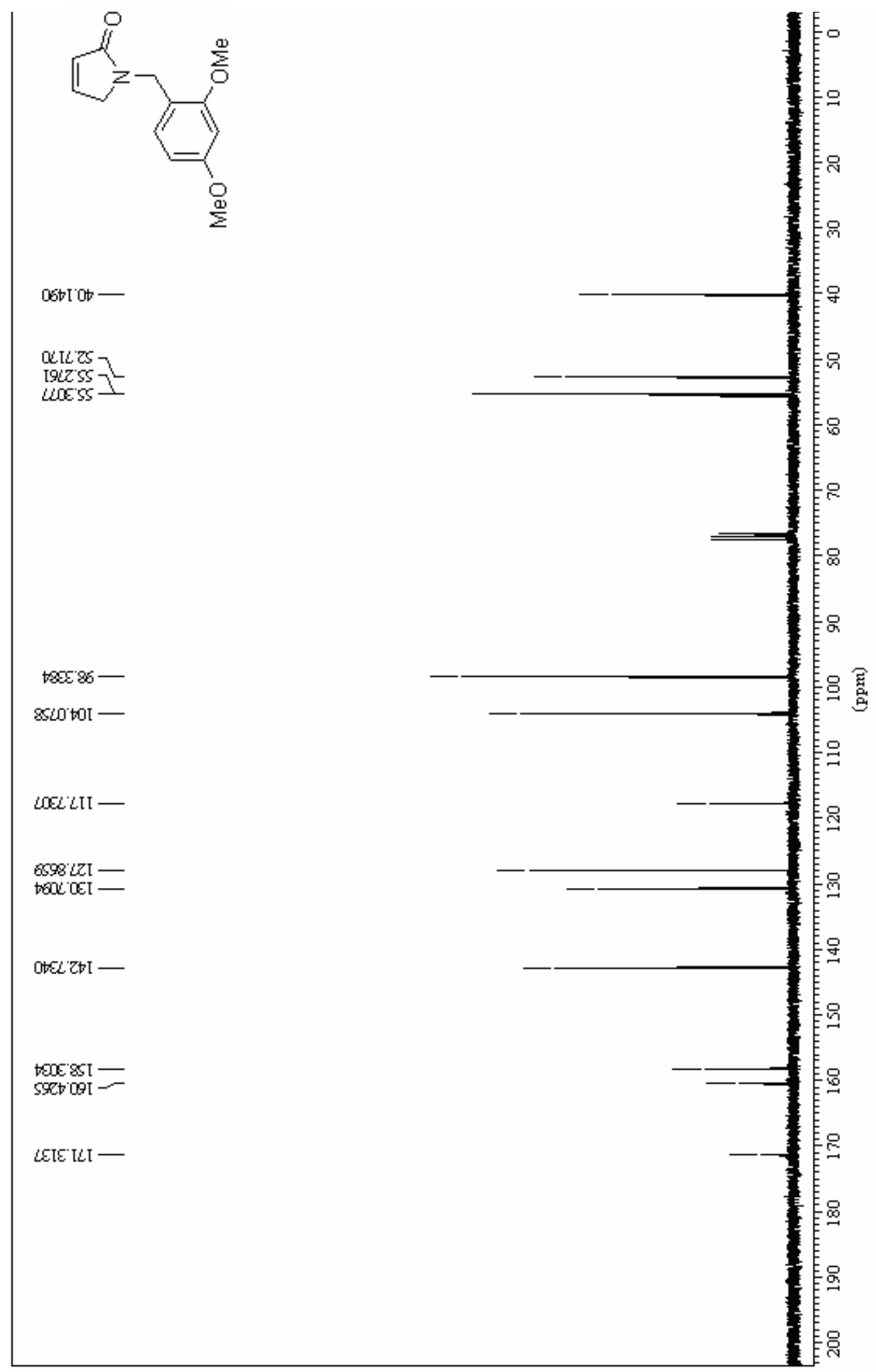


Ethyl $\quad\left(1 R^{*}, 5 S^{*}, 6 R^{*}\right)-3-(2,4-d i m e t h o x y b e n z y l)-2-o x o-3-a z a b i c y c l o[3.1 .0]$ hexane-6-carboxylate (10-trans):

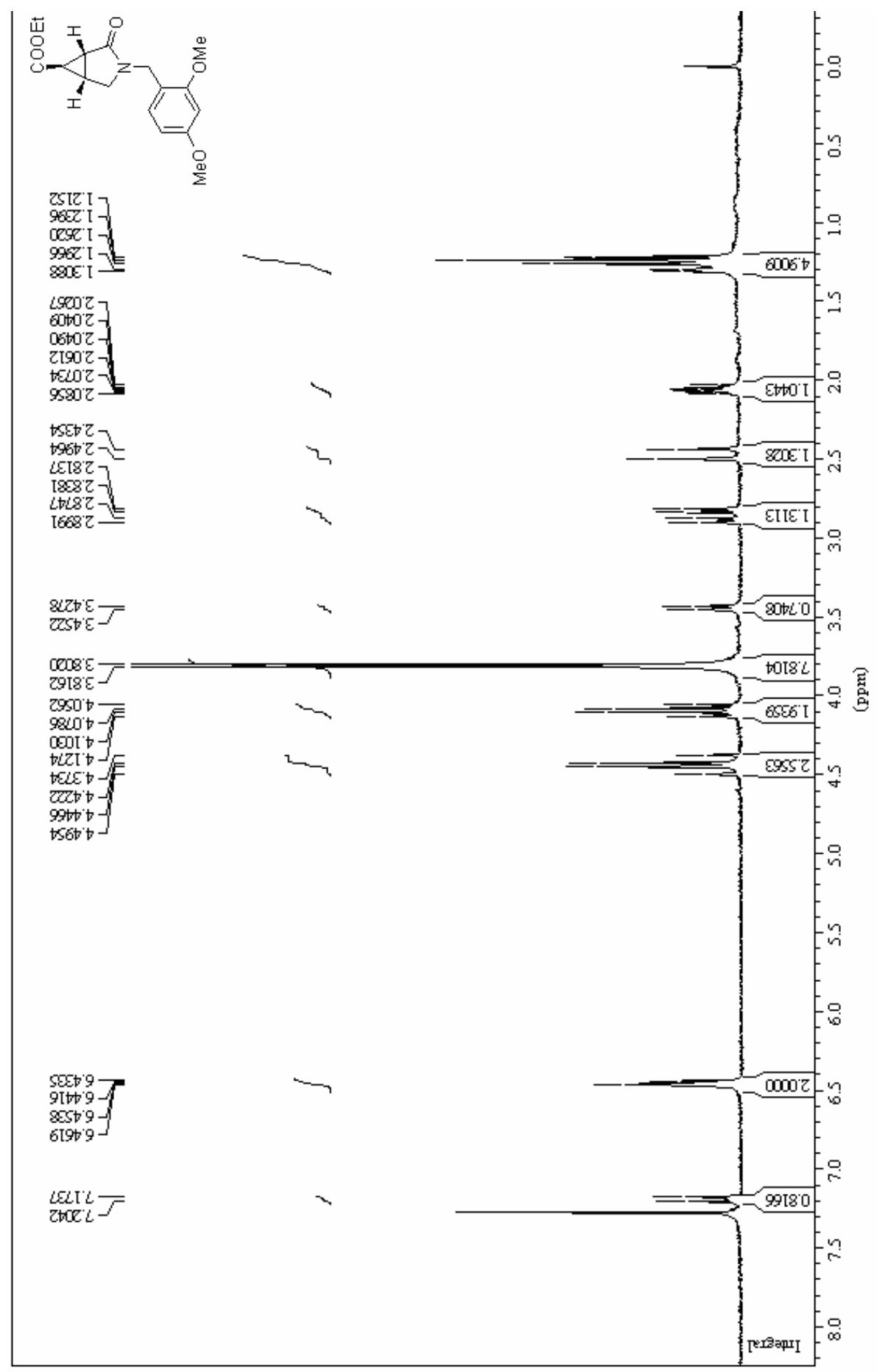




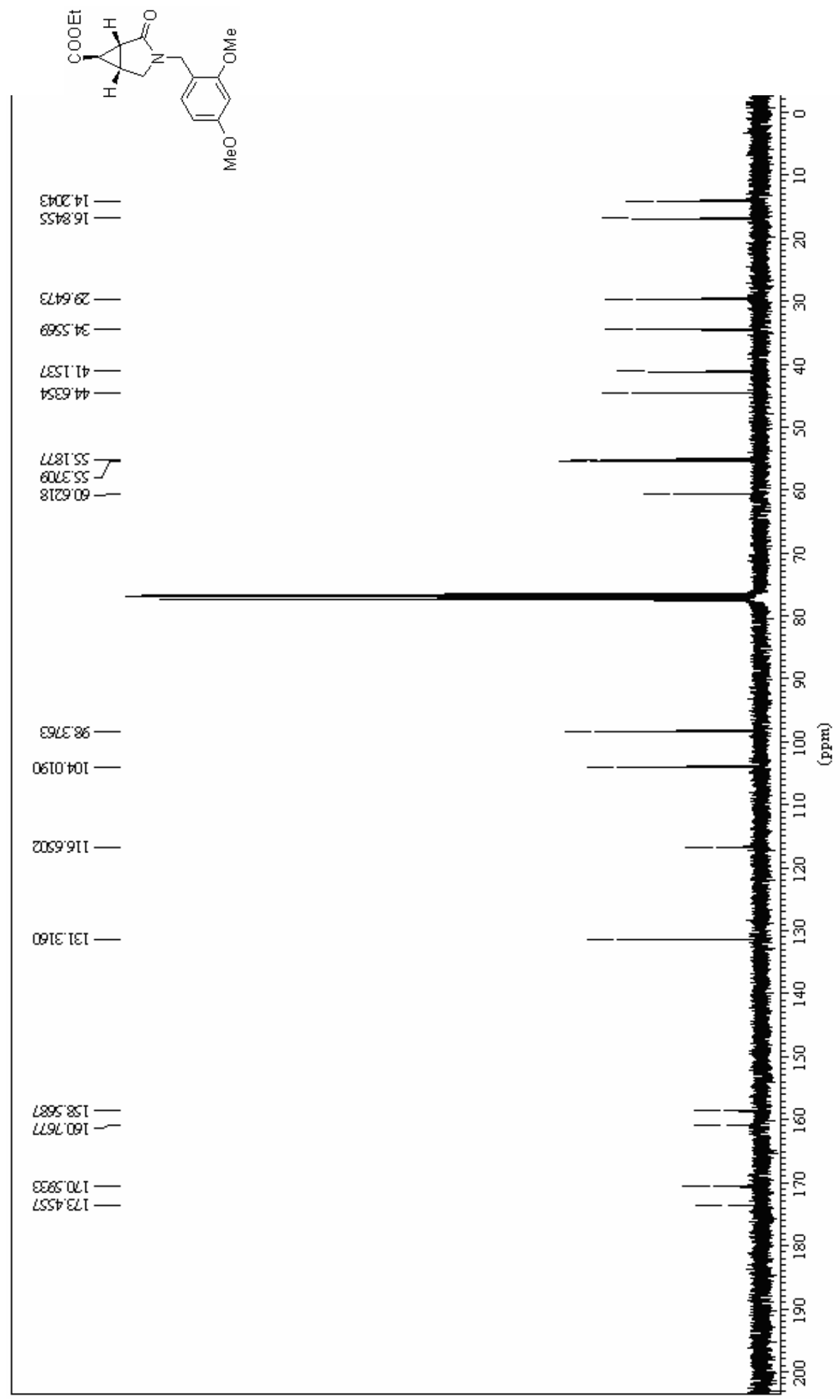


Ethyl $\quad\left(1 R^{*}, 5 S^{*}, 6 S^{*}\right)-3-(2,4-d i m e t h o x y b e n z y l)-2-o x o-3-a z a b i c y c l o[3.1 .0]$ hexane-6-carboxylate (10-cis):

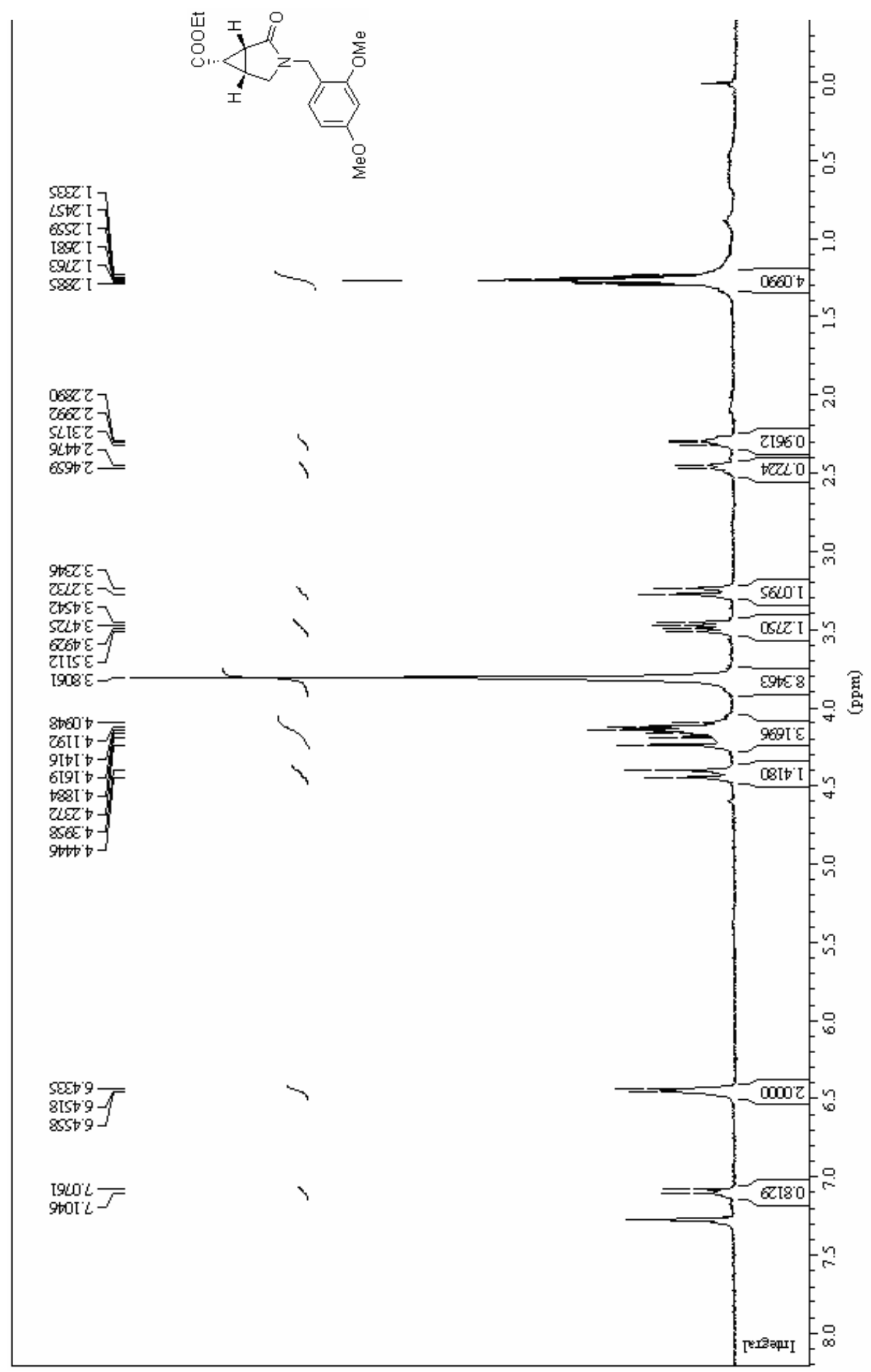




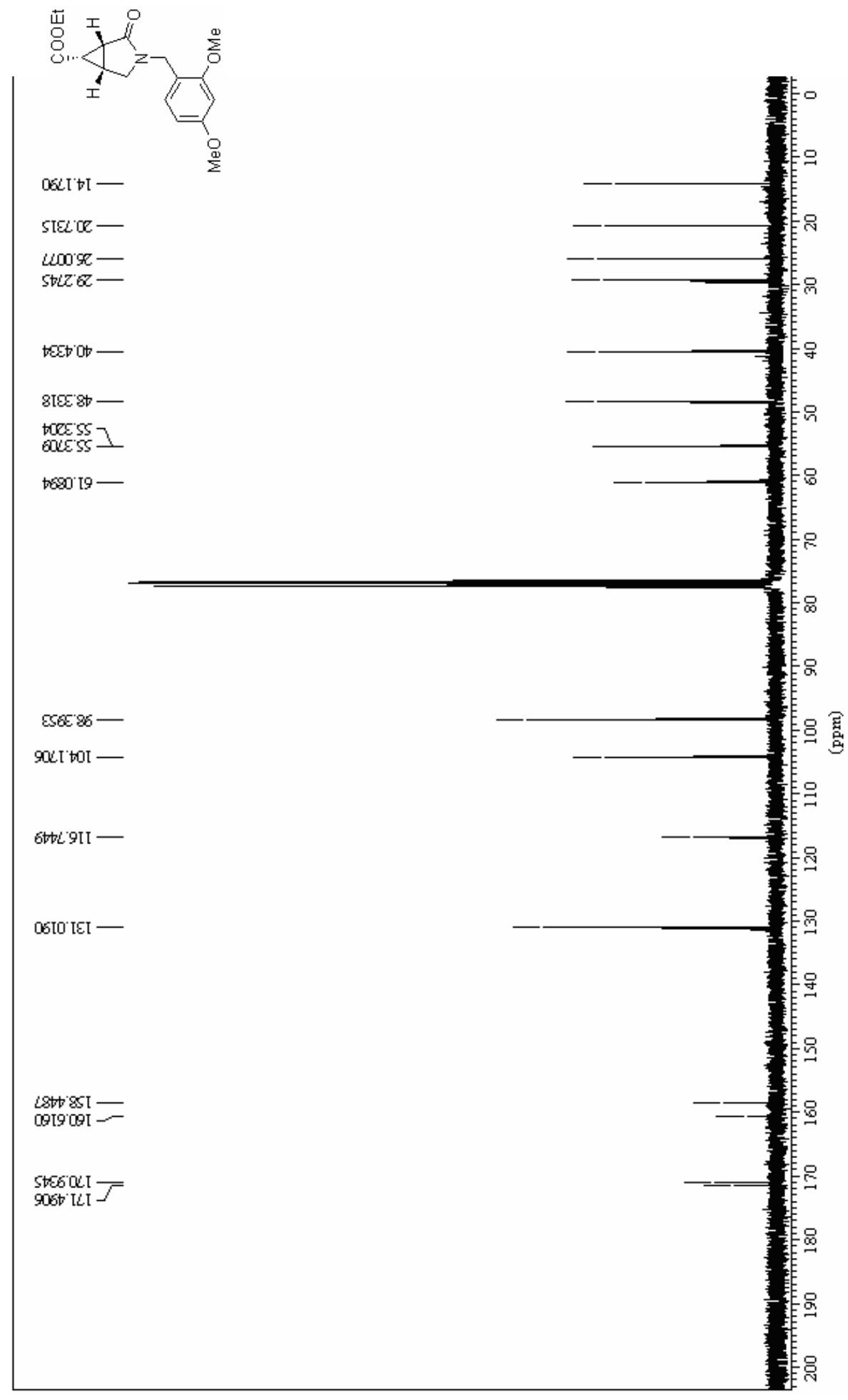

S20 
1-(2,4-dimethoxybenzyl)-3,6-dihydropyridin-2(1H)-one (2):

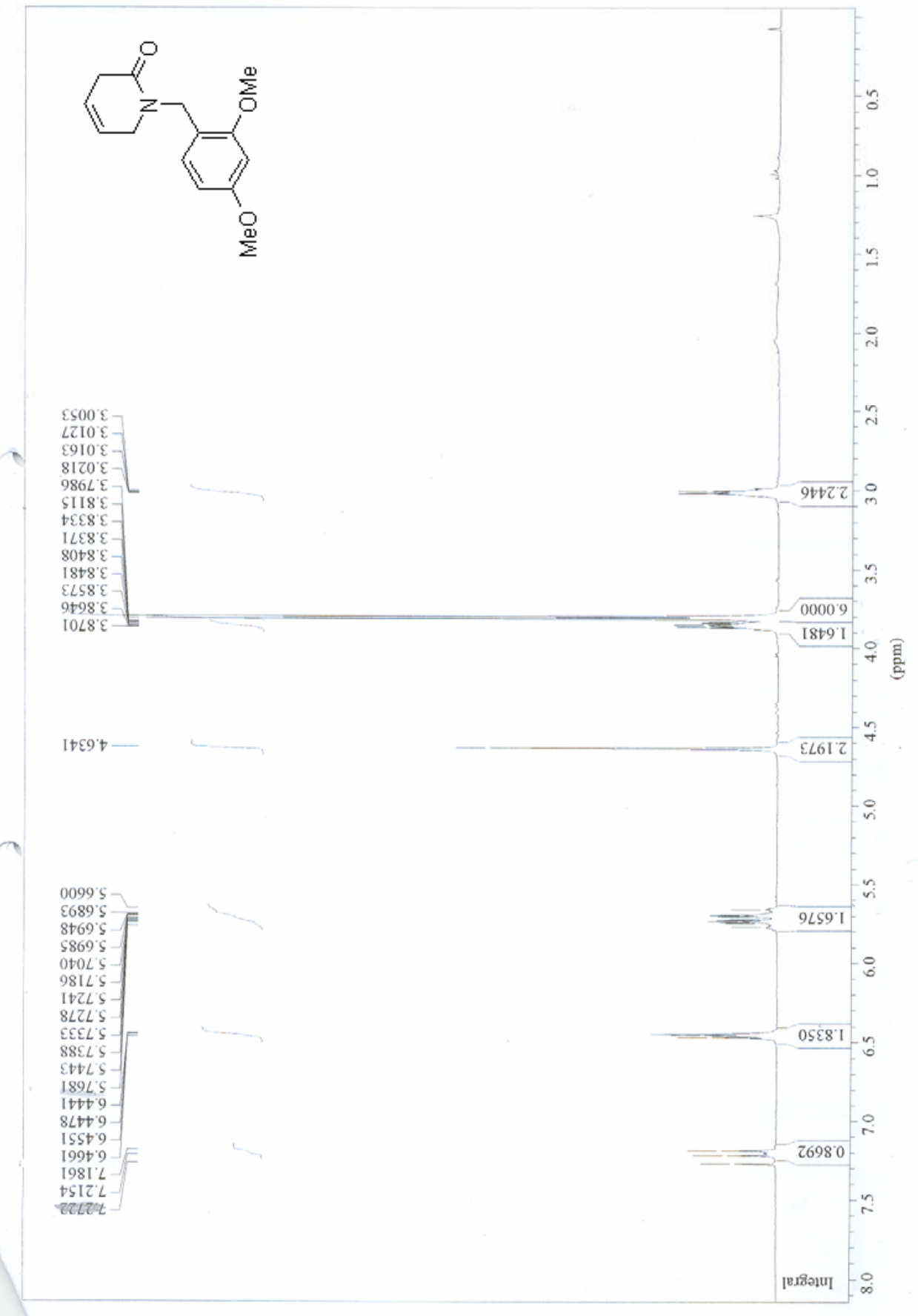




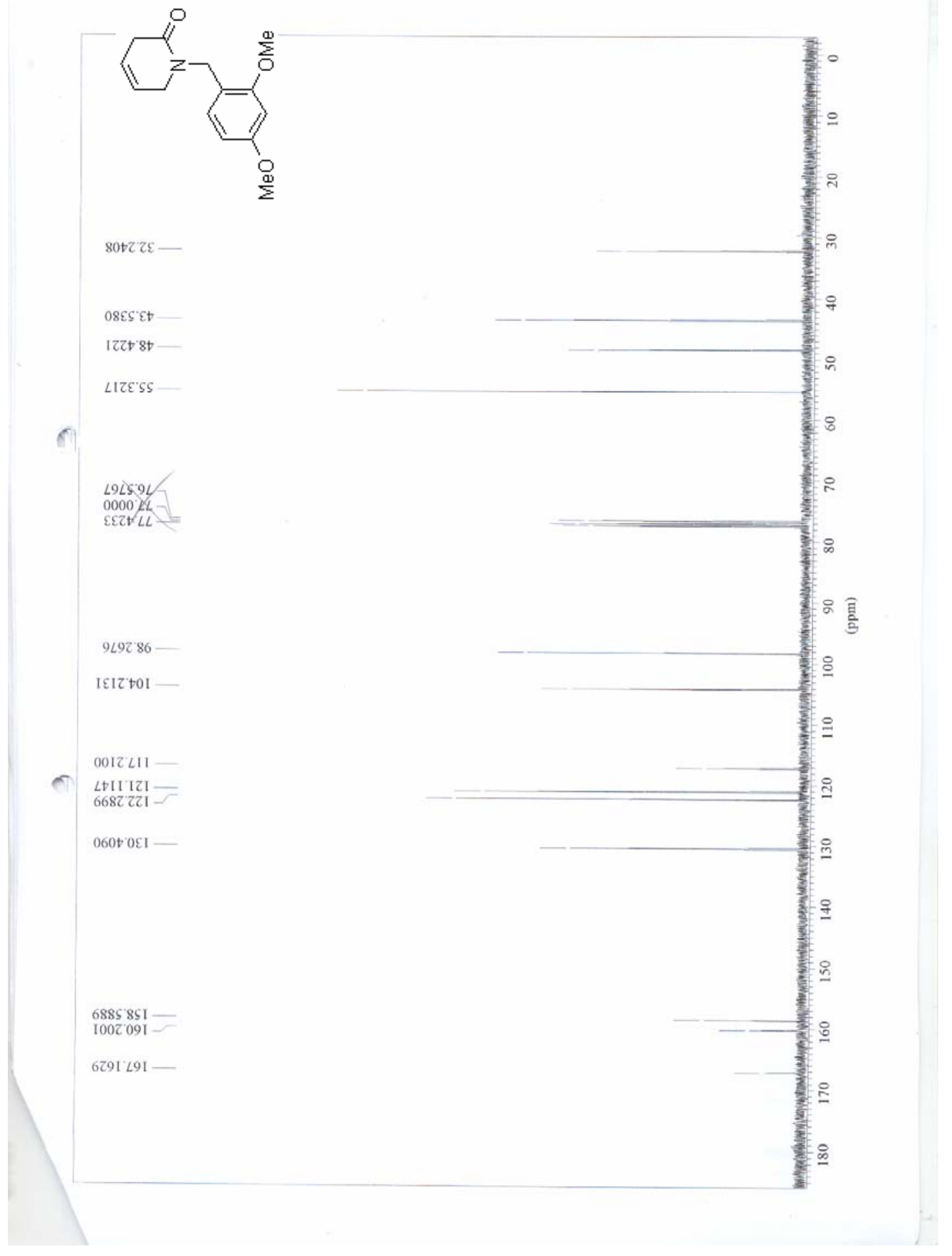


1-(2,4-dimethoxybenzyl)-5,6-dihydropyridin-2(1H)-one (3):

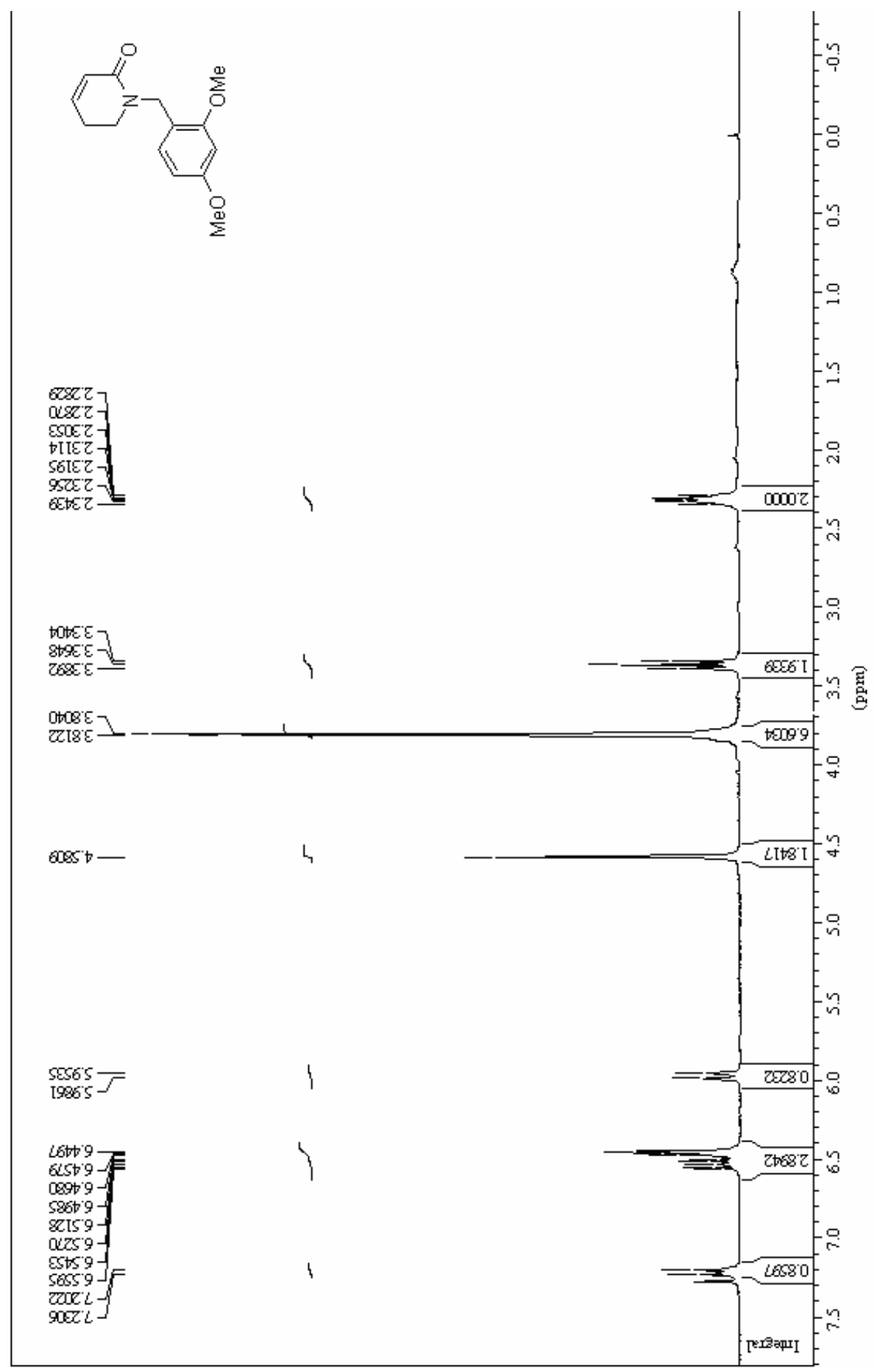




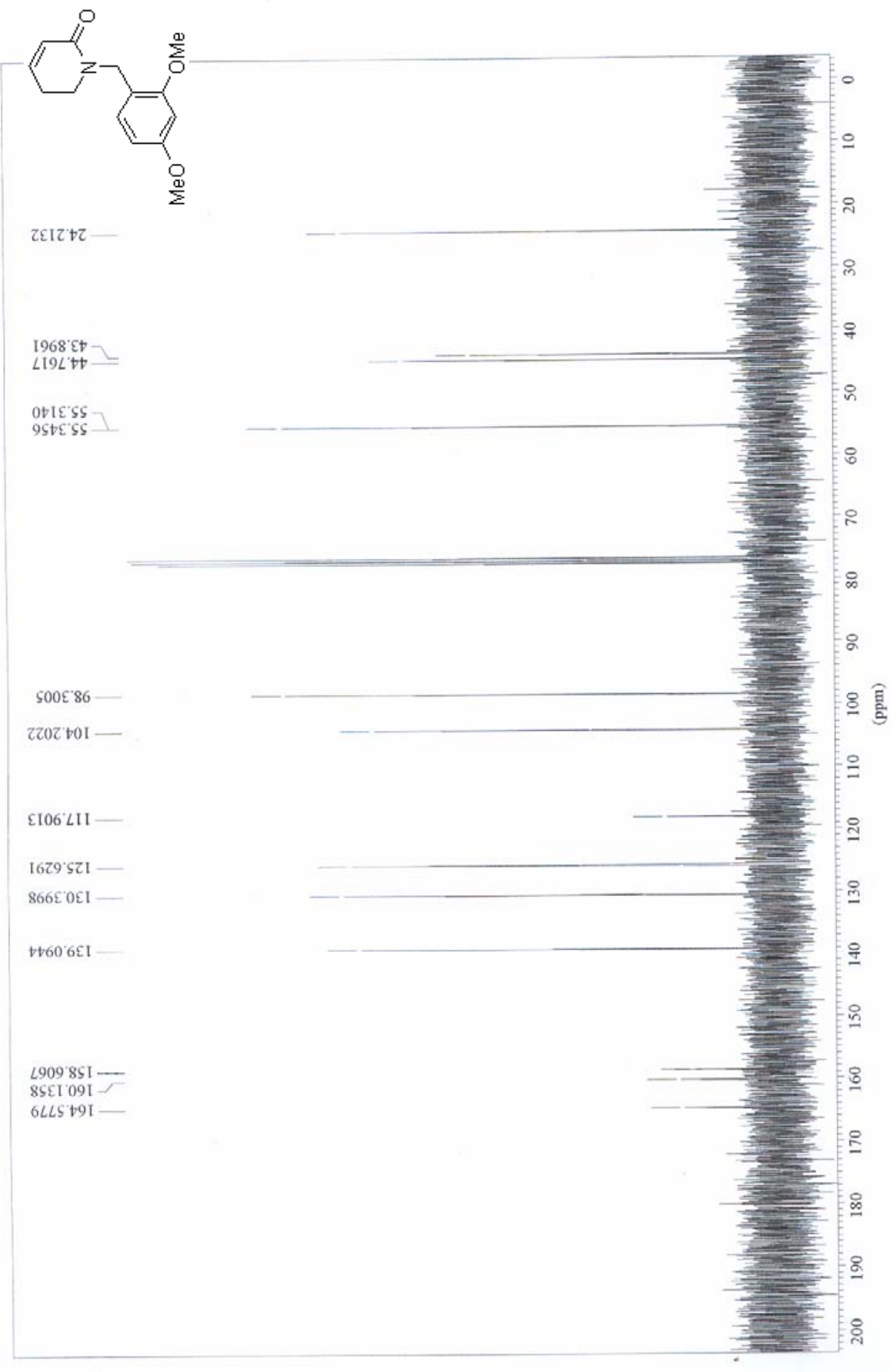


1-(2,4-dimethoxybenzyl)-3,4-dihydropyridin-2(1H)-one (4):

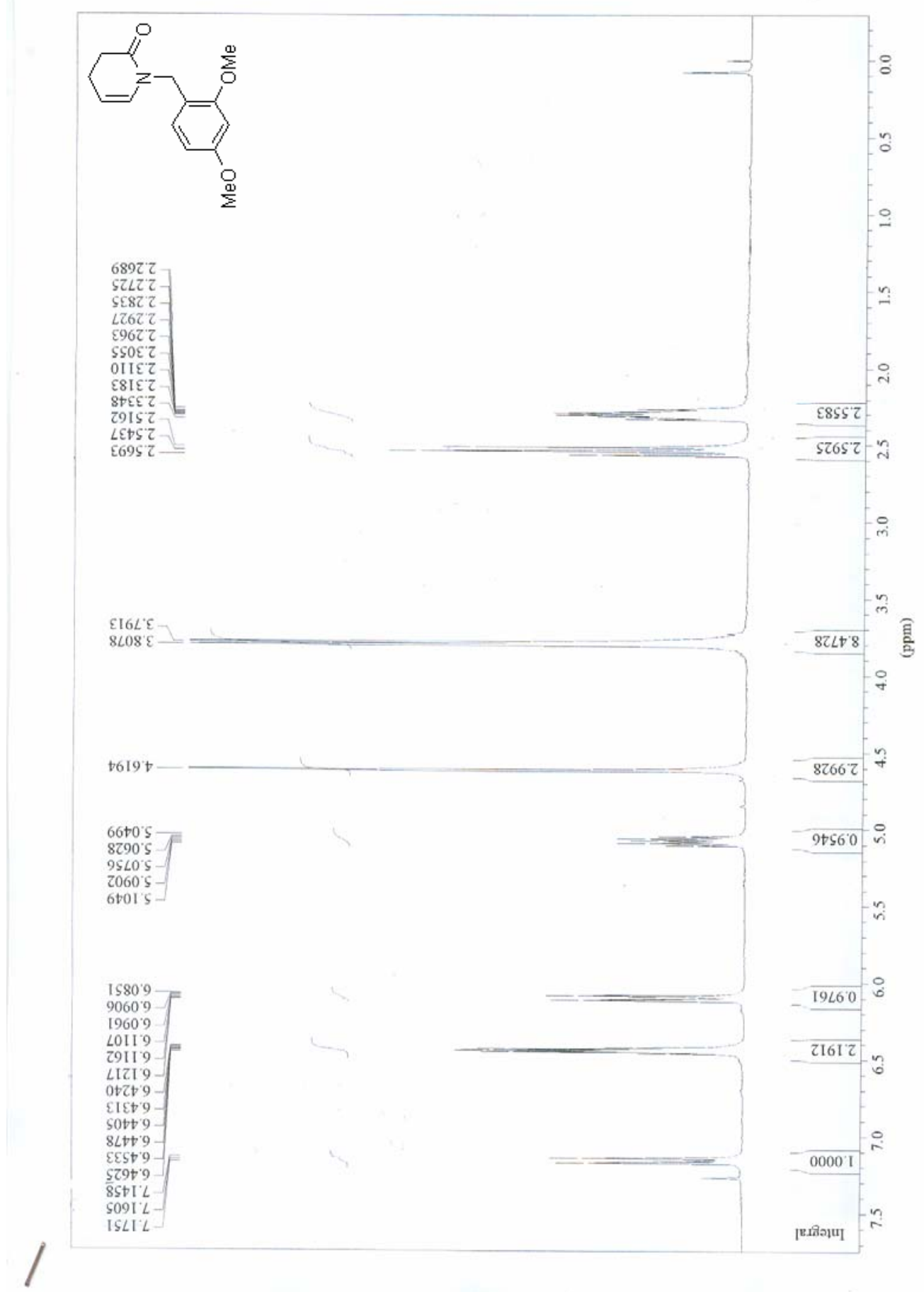




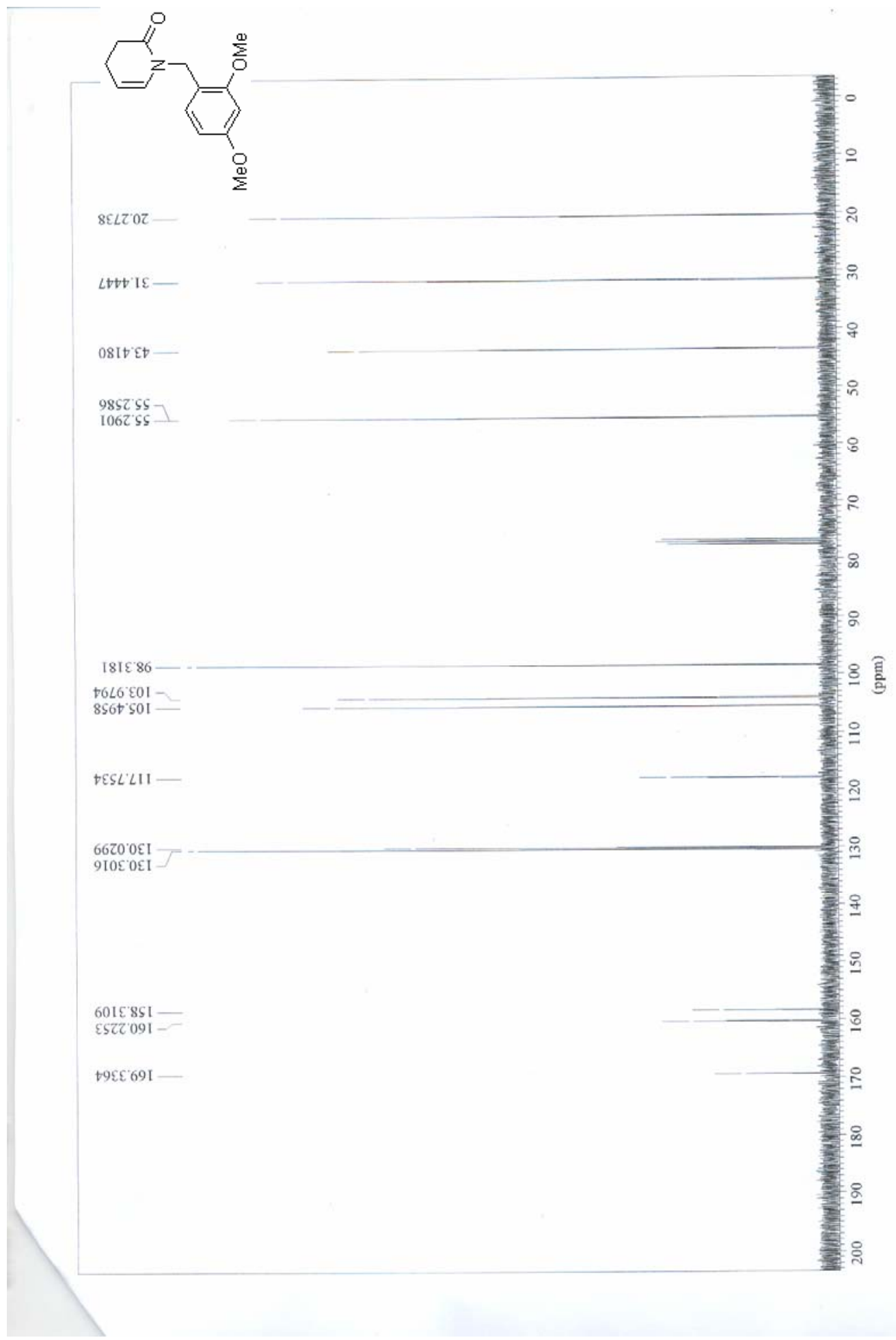

S26 
Ethyl $\quad\left(1 S^{*}, 6 S^{*}, 7 S^{*}\right)-2-(2,4-d i m e t h o x y b e n z y l)-3-o x o-2-a z a b i c y c l o[4.1 .0]$ heptane-7-carboxylate (6-trans):

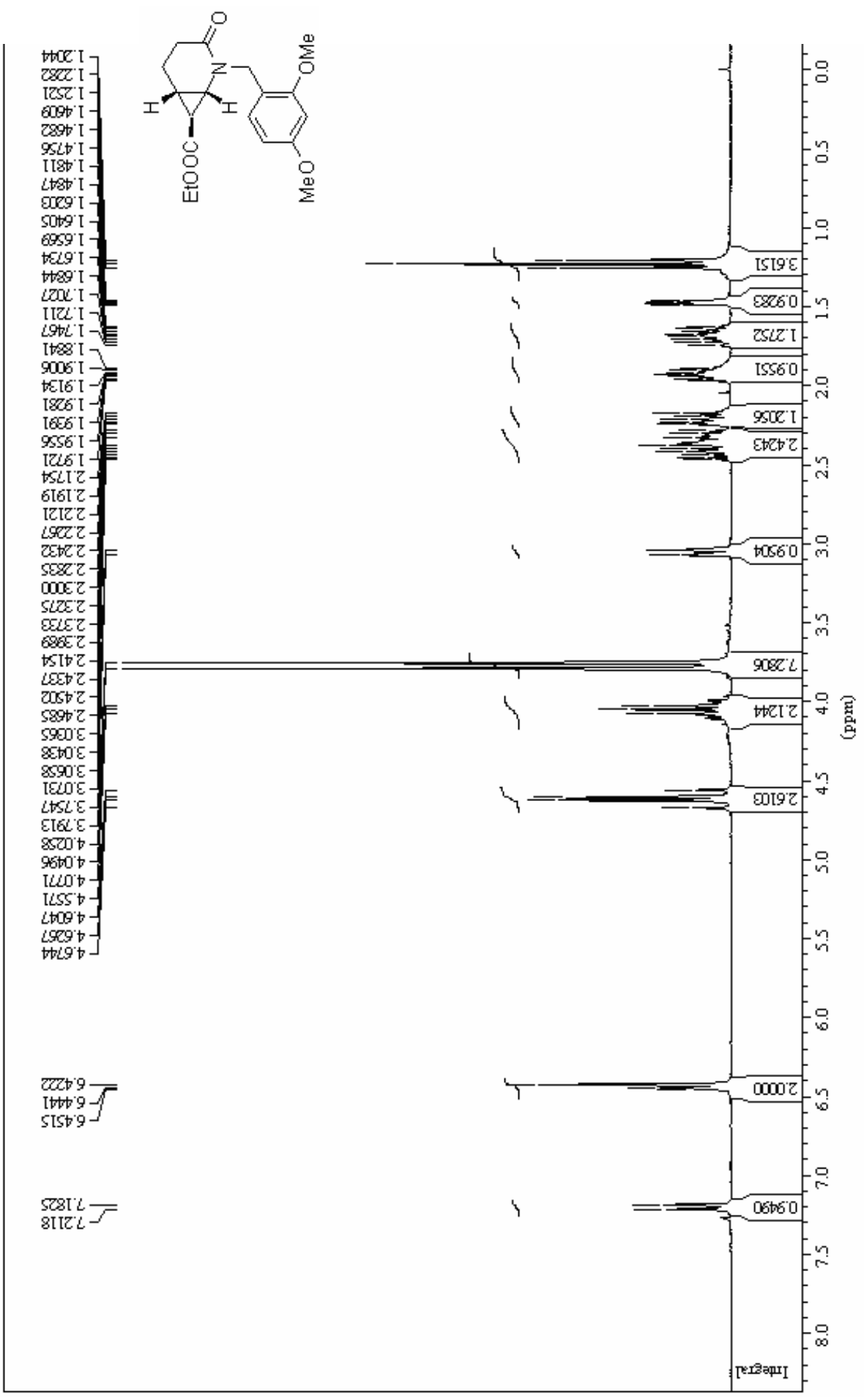




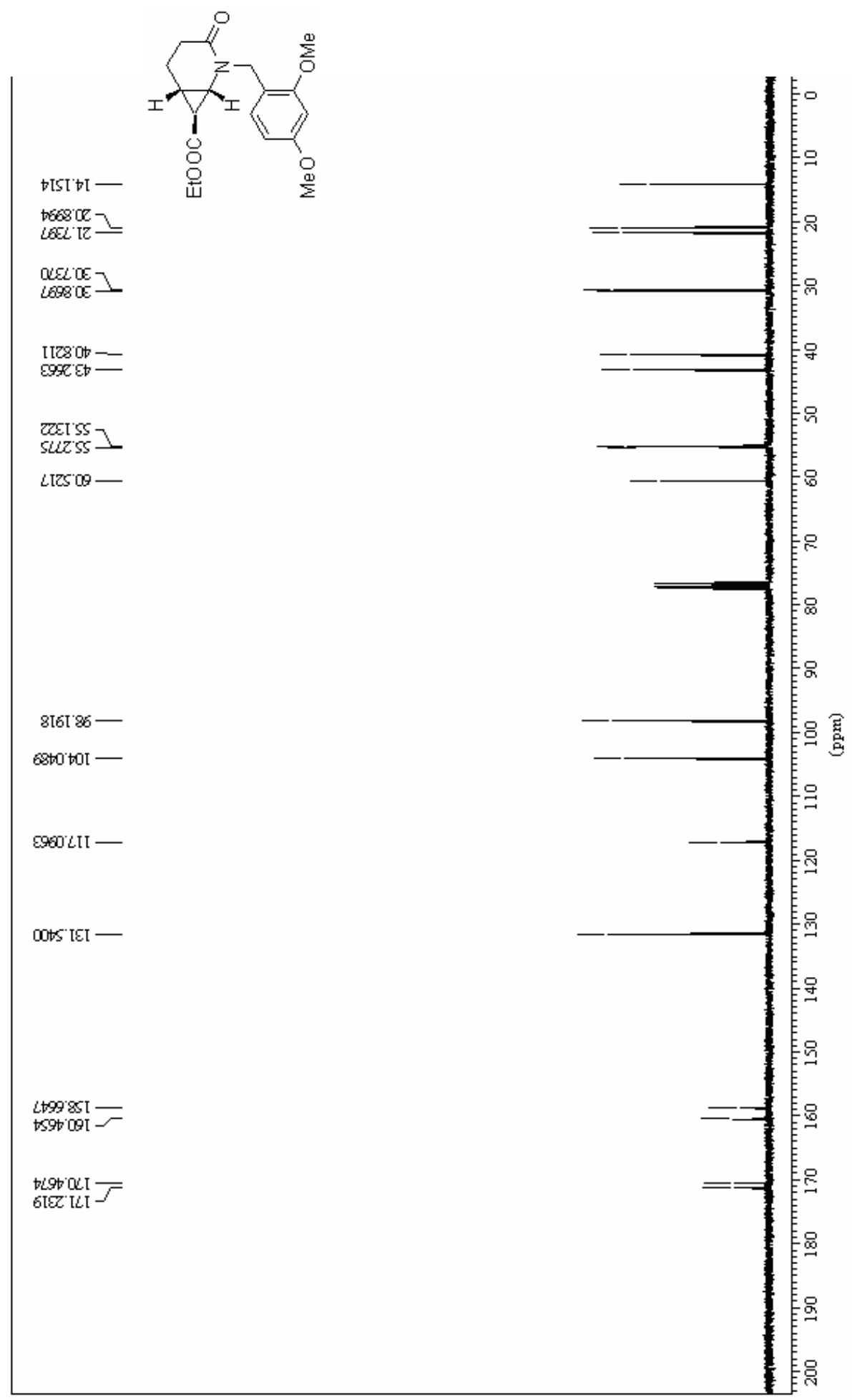


Ethyl $\quad\left(1 S^{*}, 6 S^{*}, 7 R^{*}\right)-2-(2,4-d i m e t h o x y b e n z y l)-3-o x o-2-a z a b i c y c l o[4.1 .0]$ heptane-7-carboxylate (6-cis):

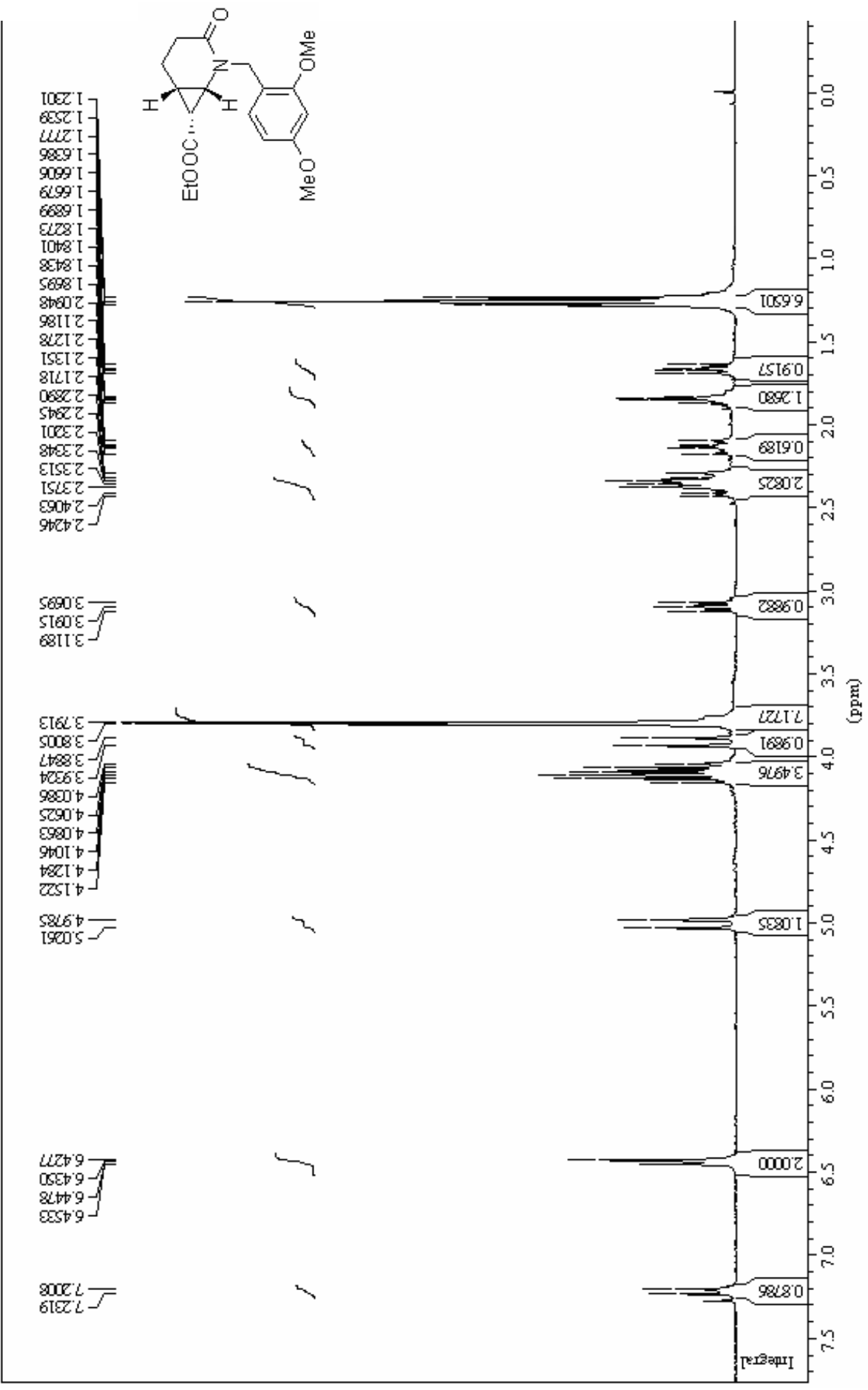




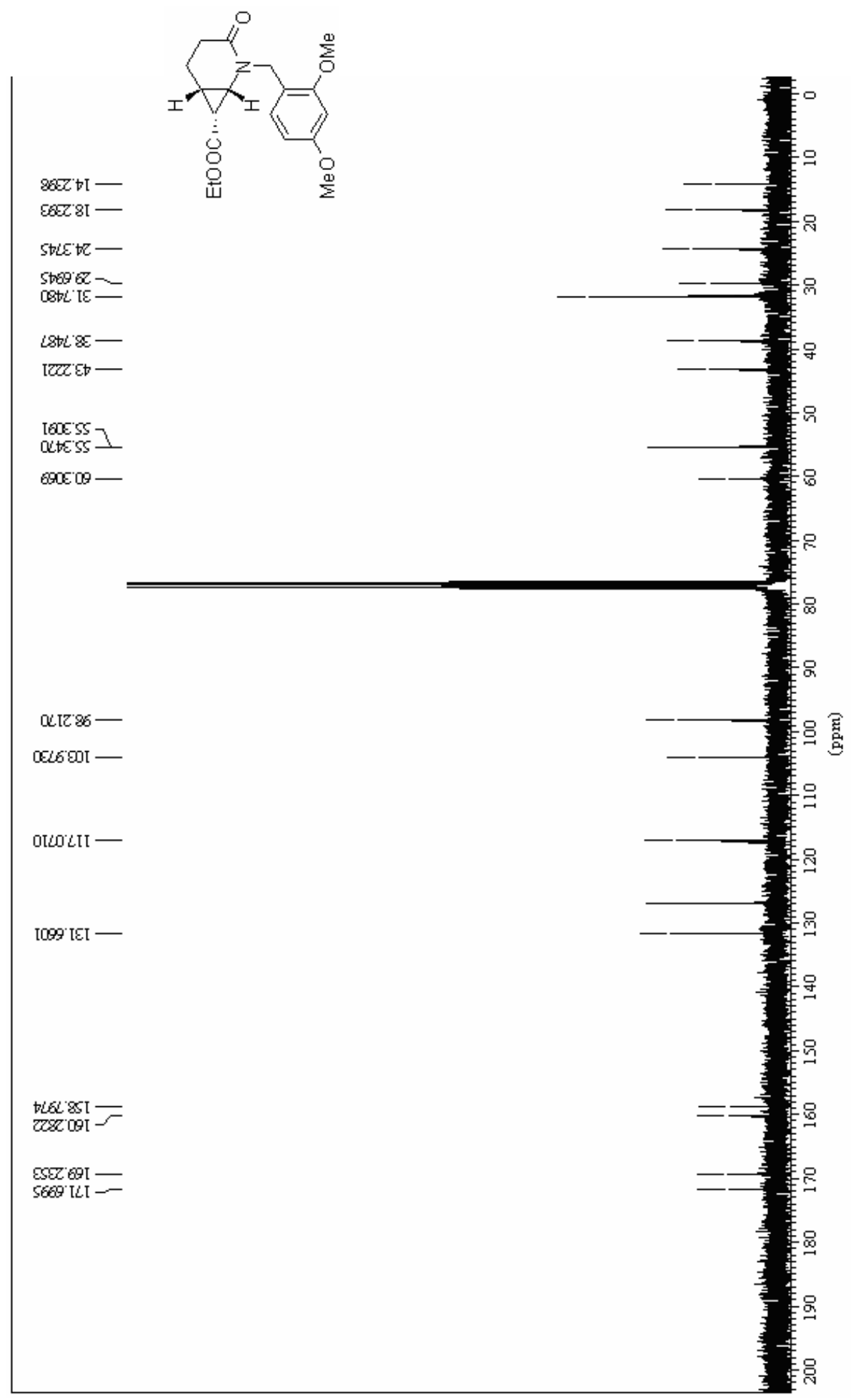

S30 


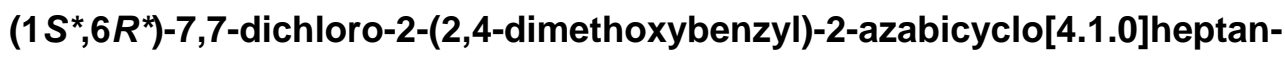
3-one (5):

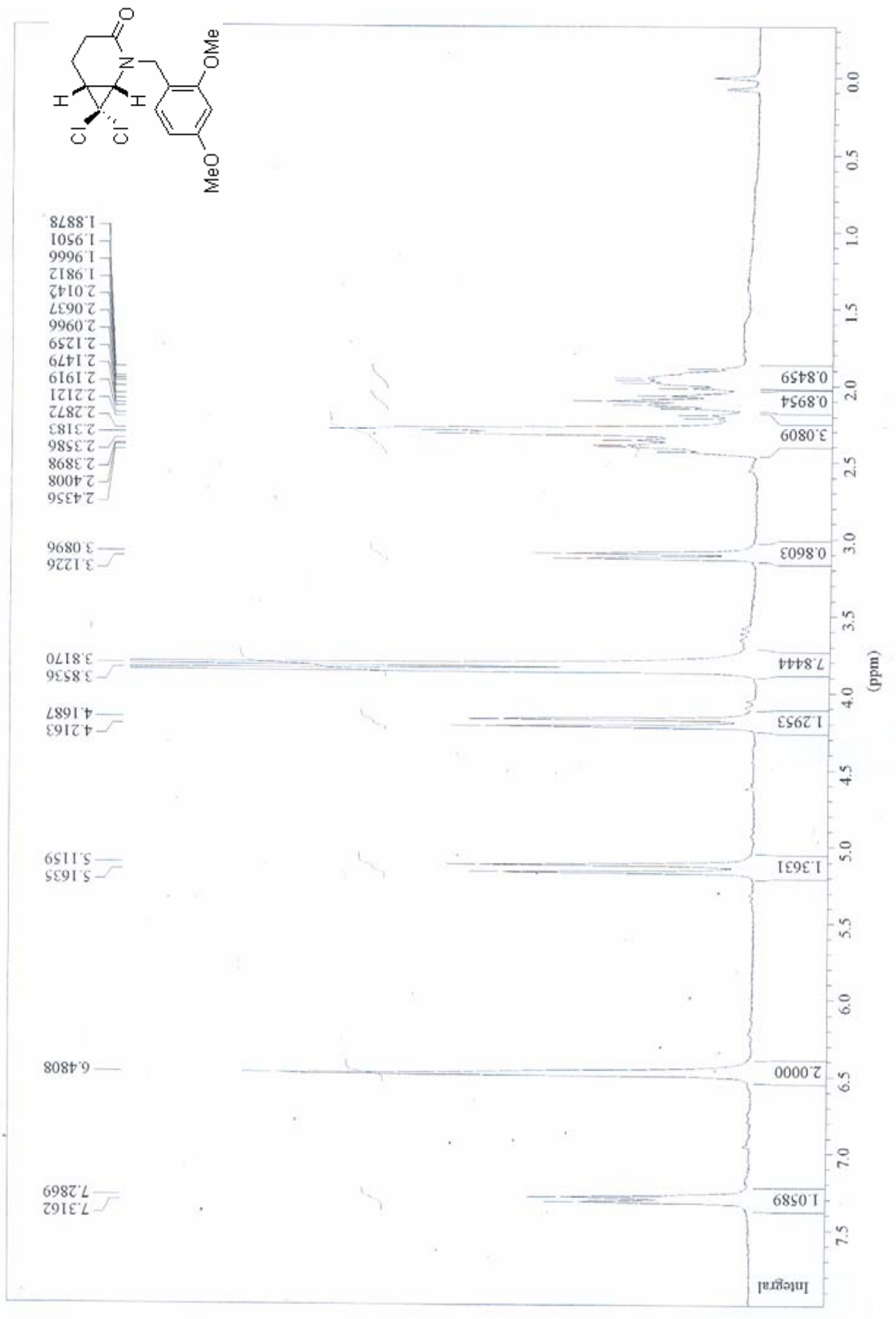




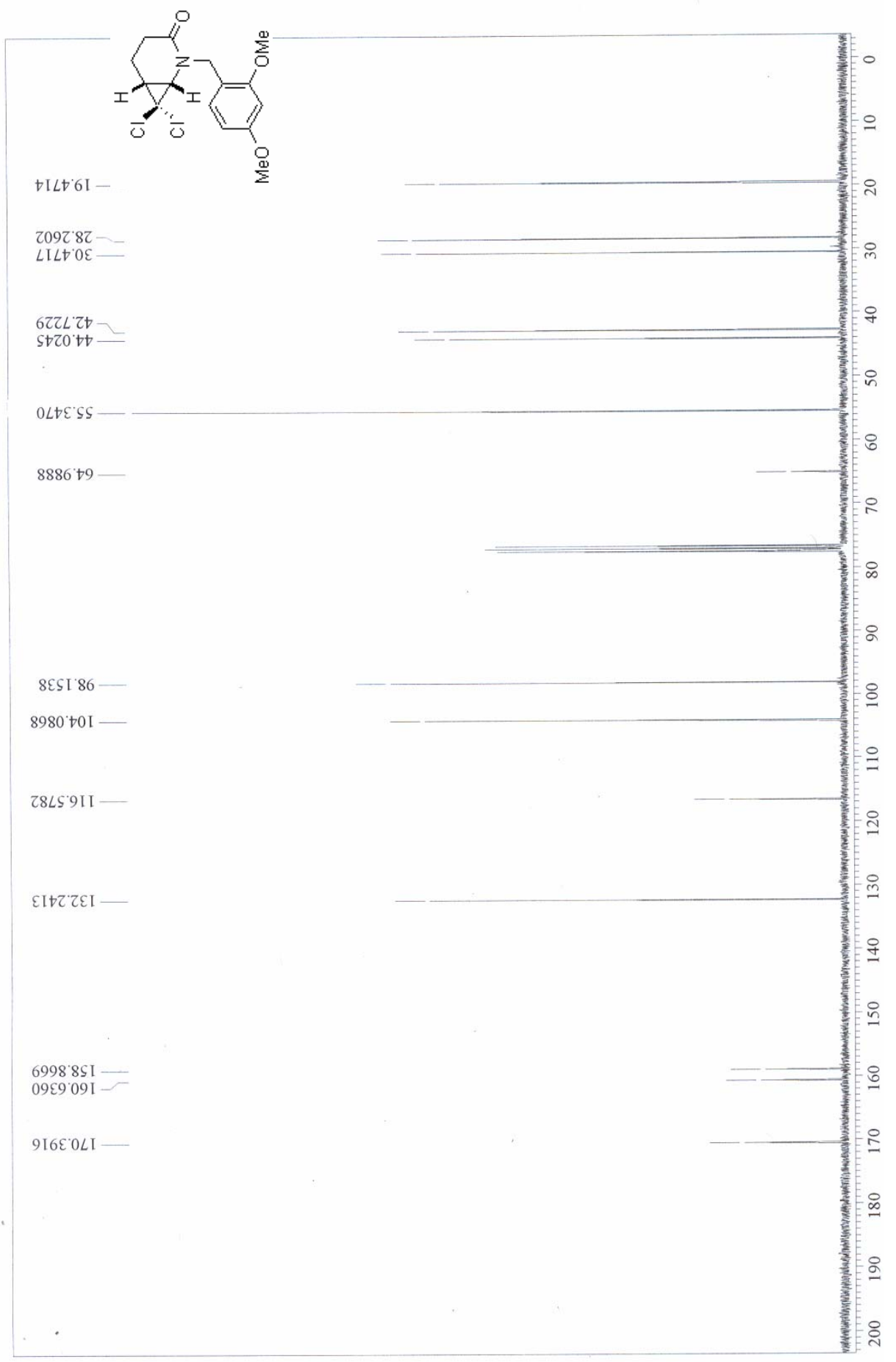


1-(2,4-dimethoxybenzyl)-1,3,4,5-tetrahydro-2H-azepin-2-one (12):

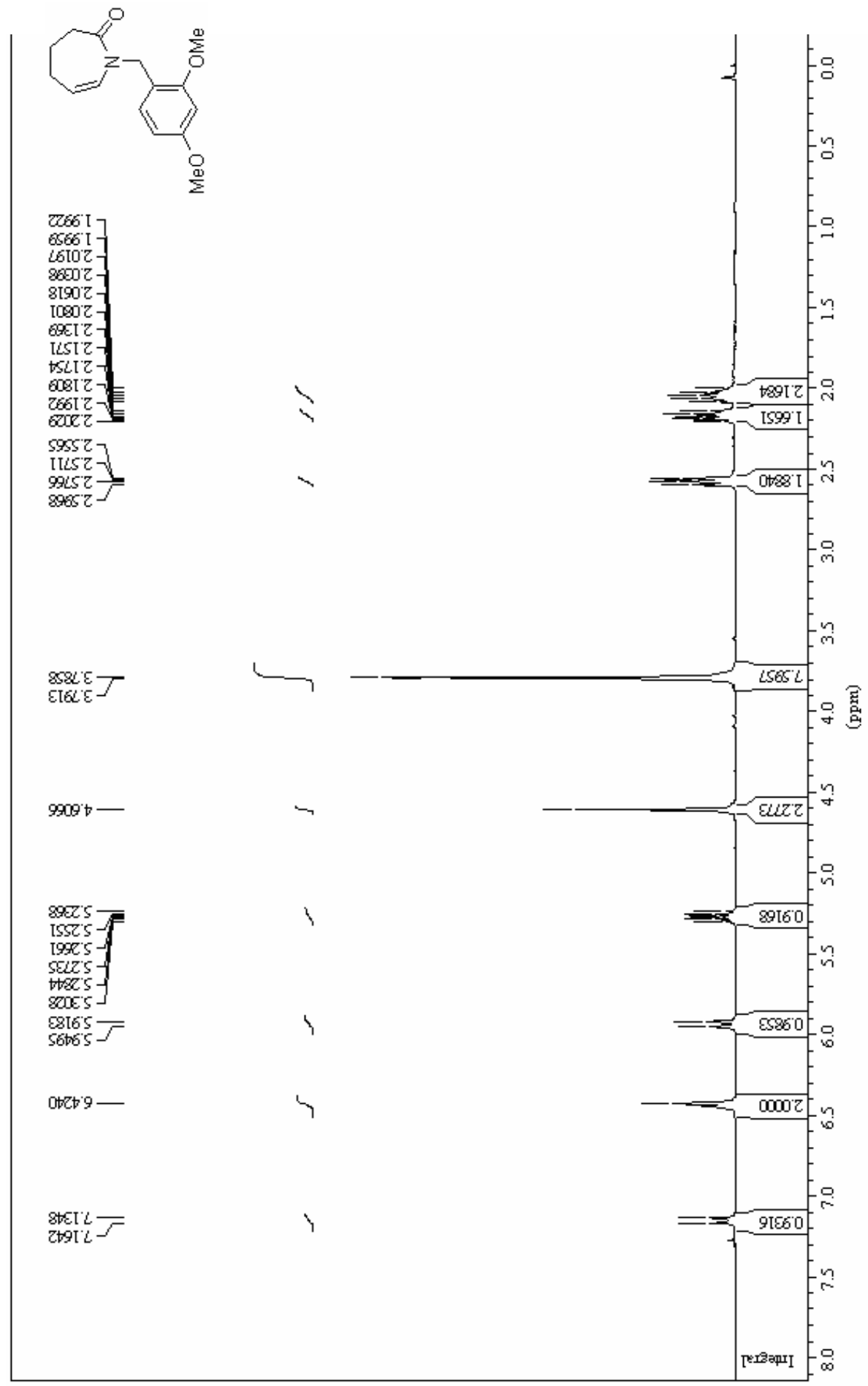




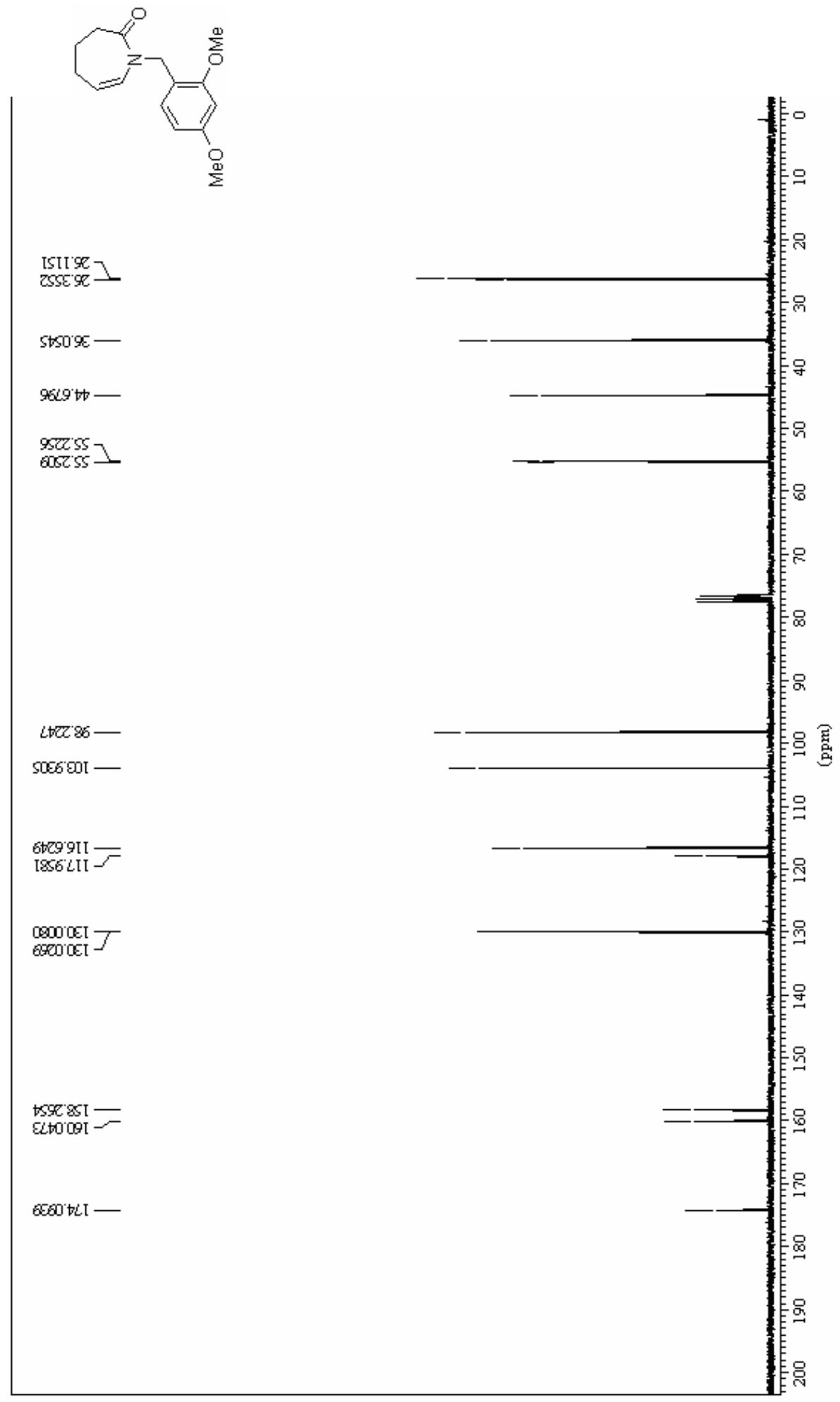


1-(2,4-dimethoxybenzyl)-1,3,6,7-tetrahydro-2H-azepin-2-one (13):

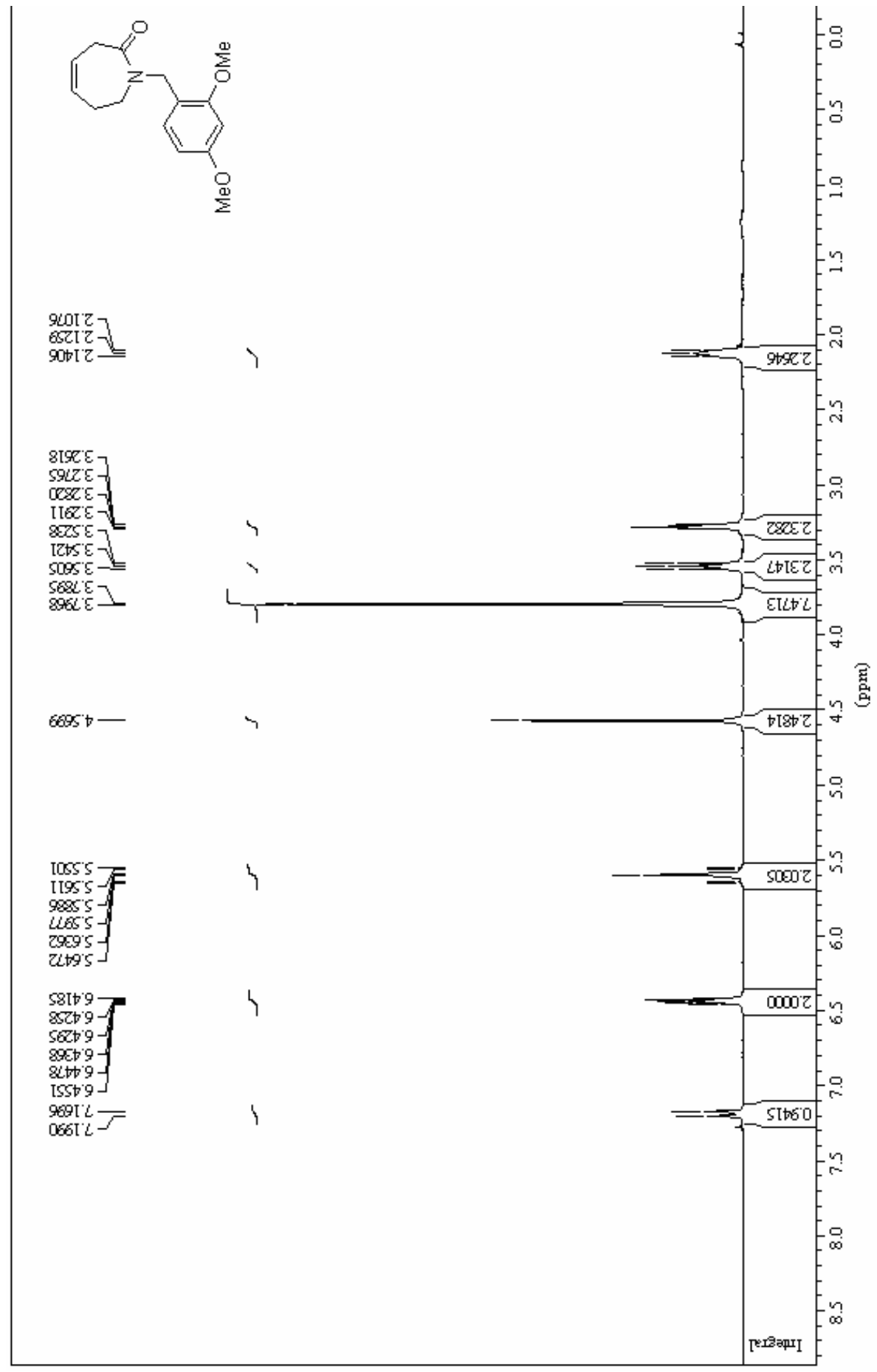




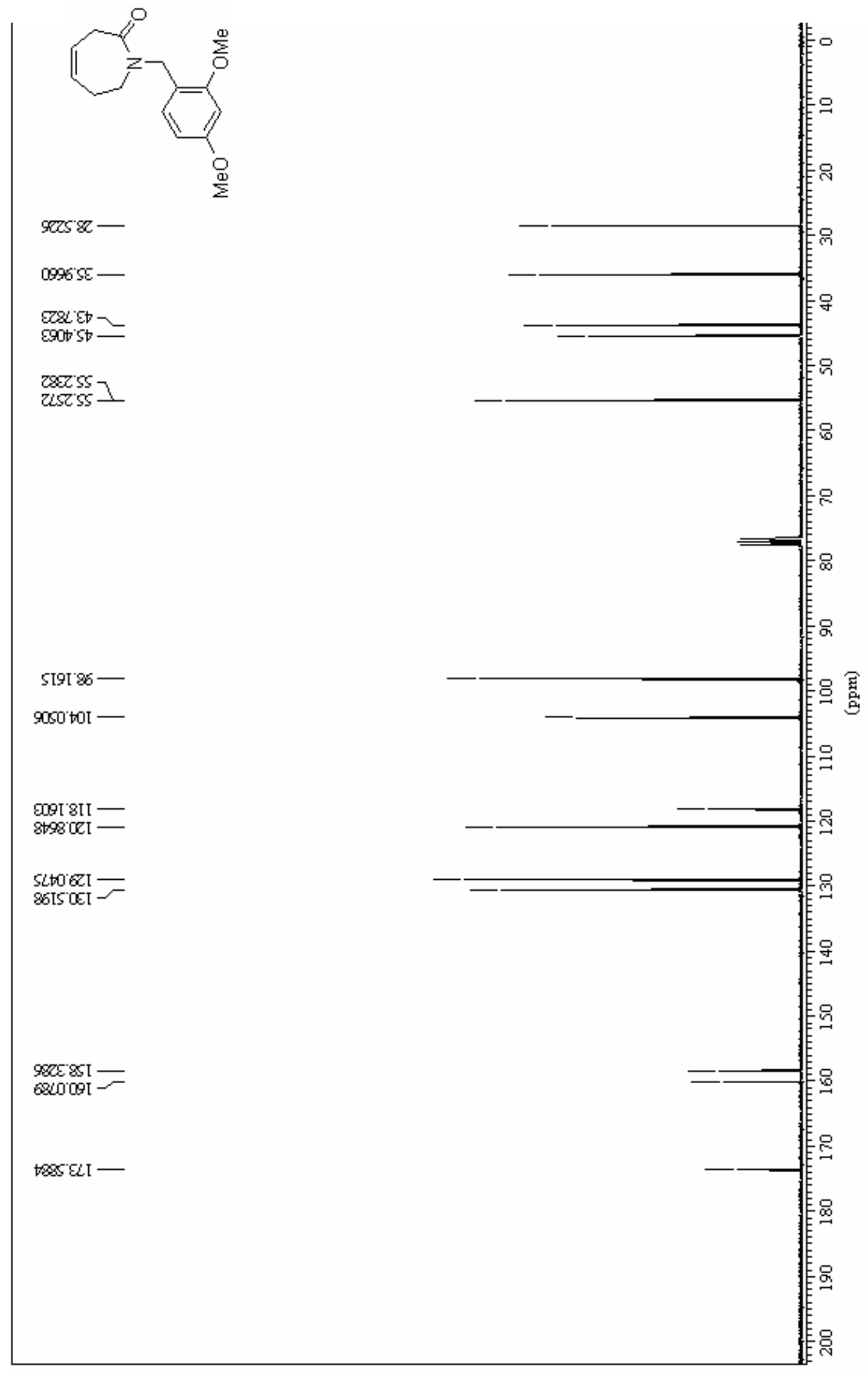


1-(2,4-dimethoxybenzyl)-1,5,6,7-tetrahydro-2H-azepin-2-one (14):

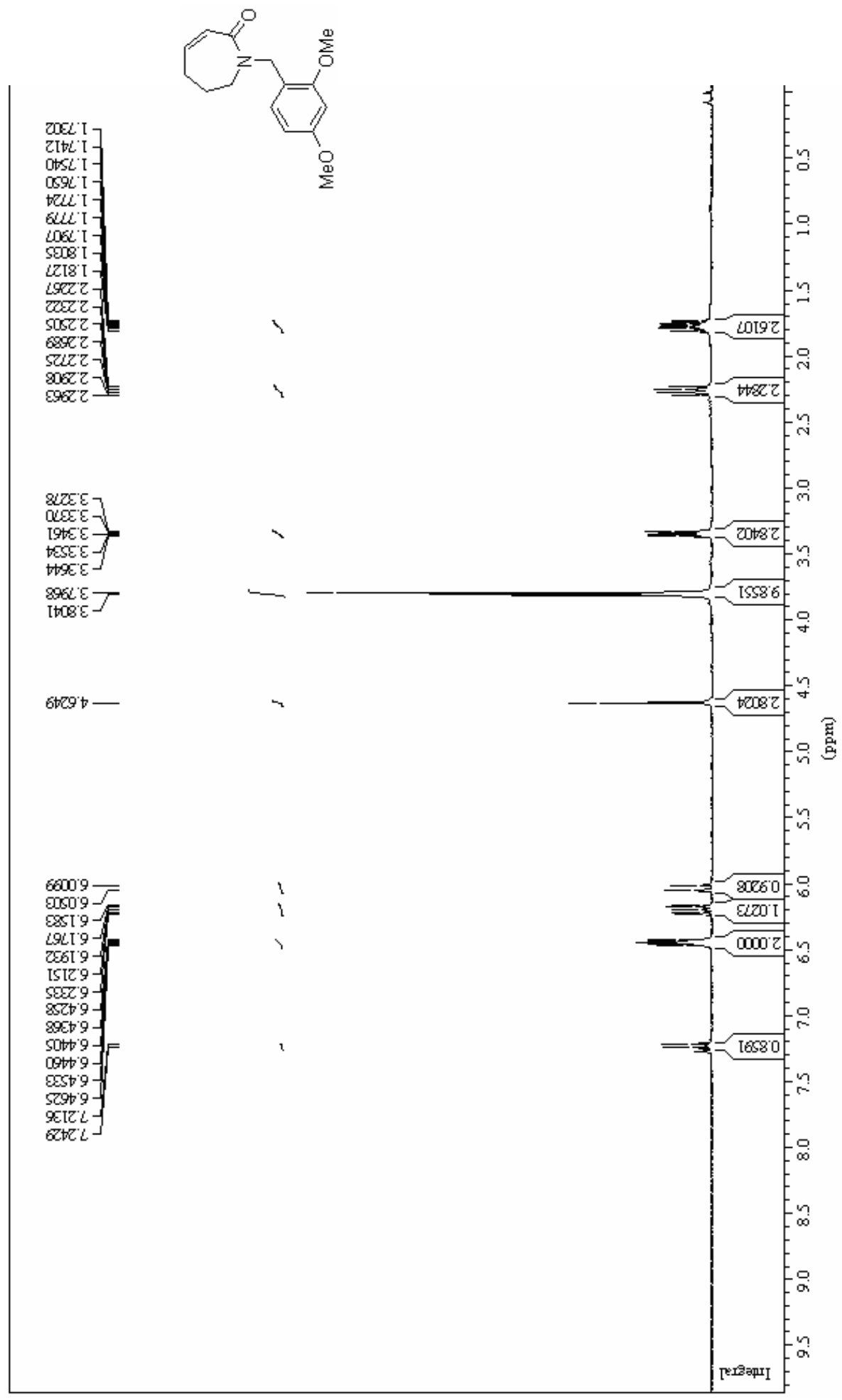




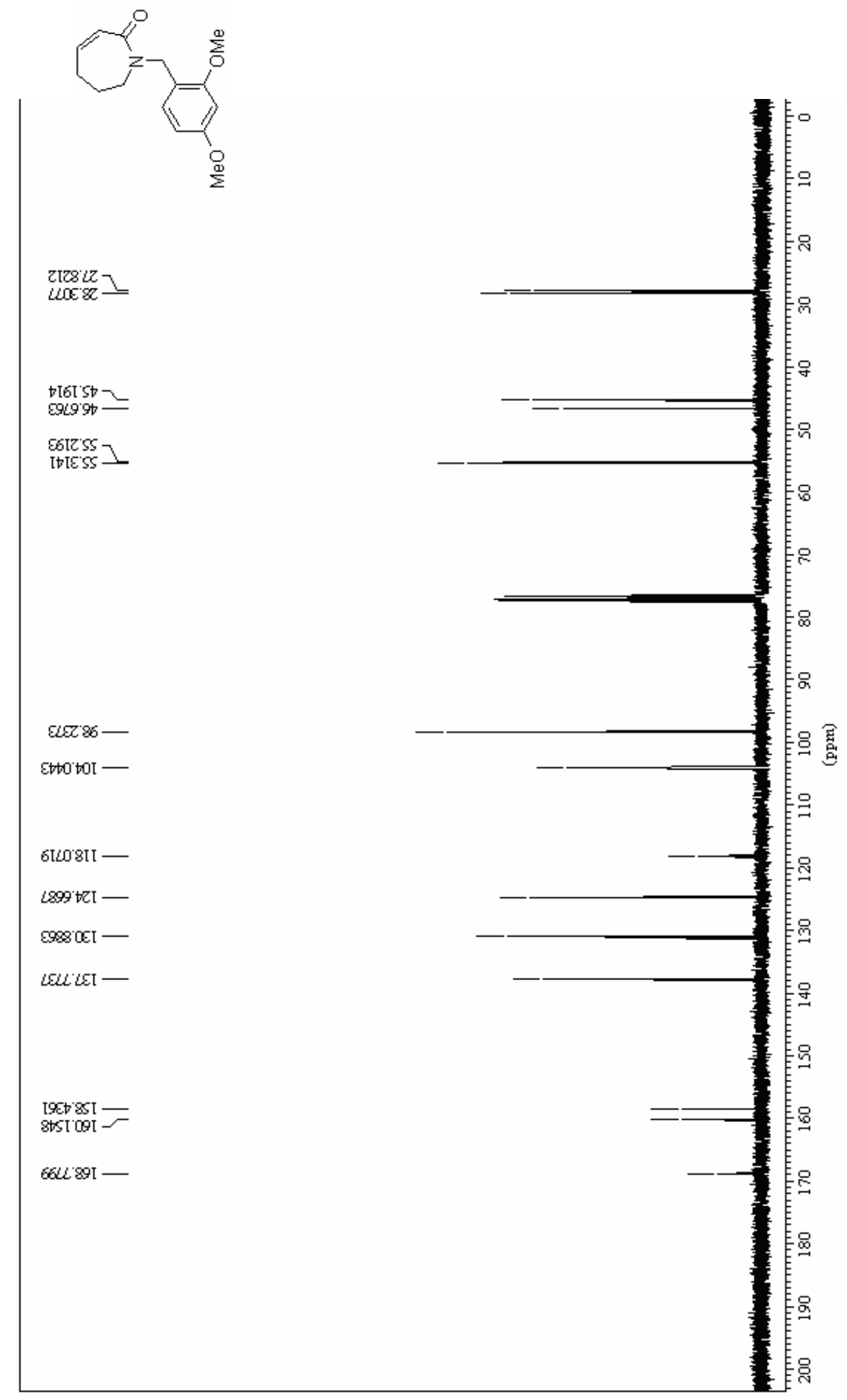

S38 
1-(2,4-dimethoxybenzyl)-1,3,4,7-tetrahydro-2H-azepin-2-one (15):

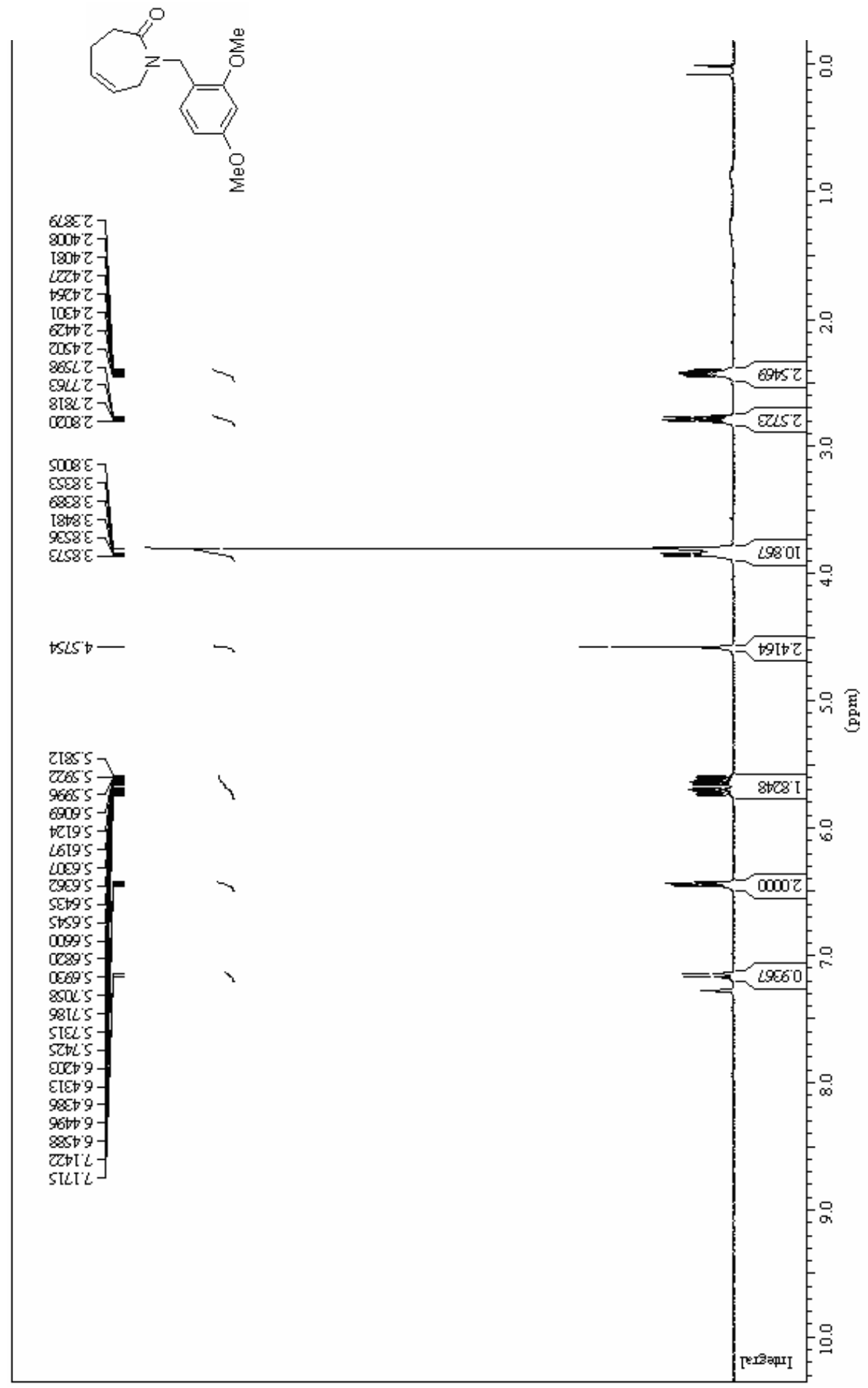




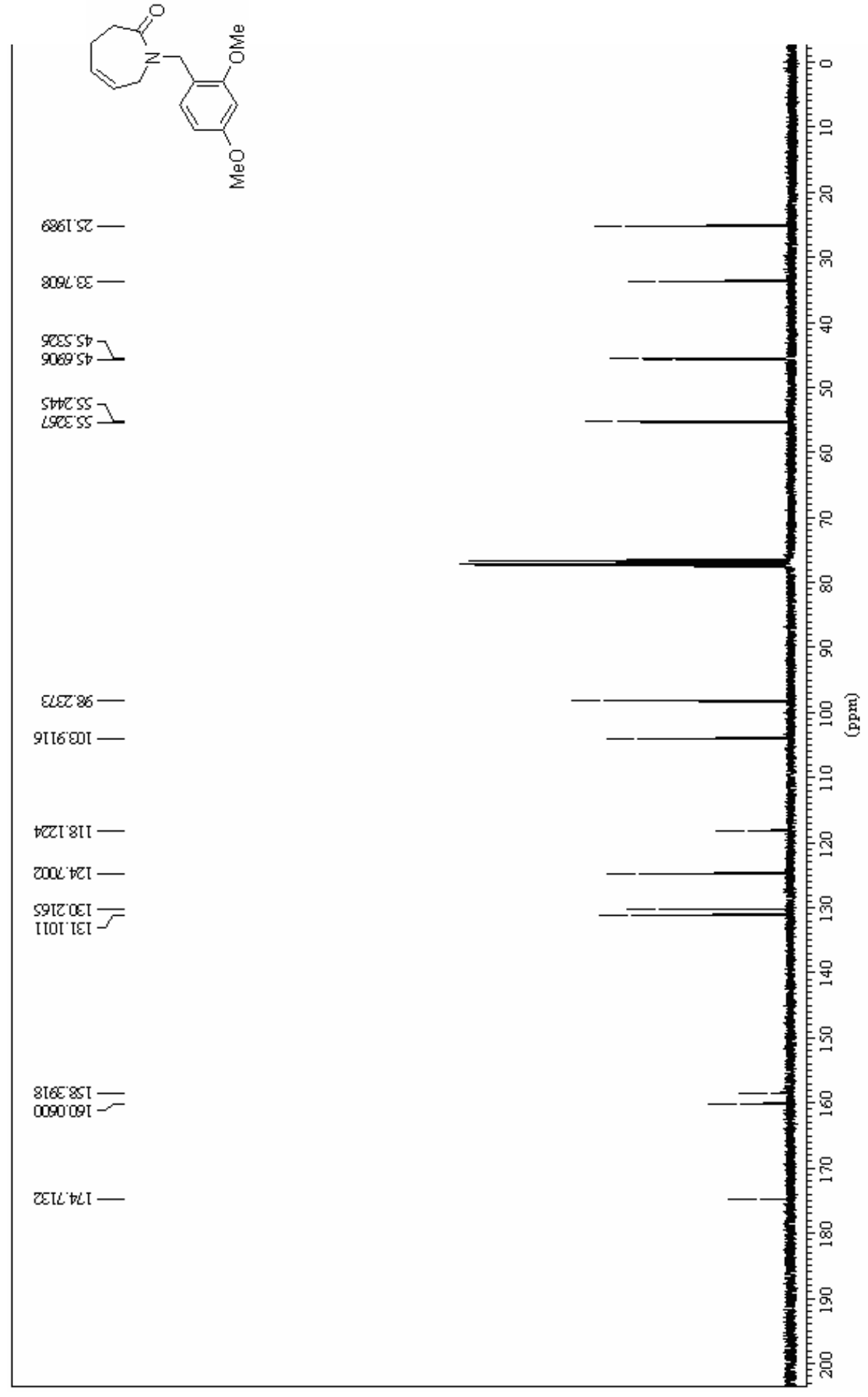

S40 
octane-8-carboxylate (16-trans):

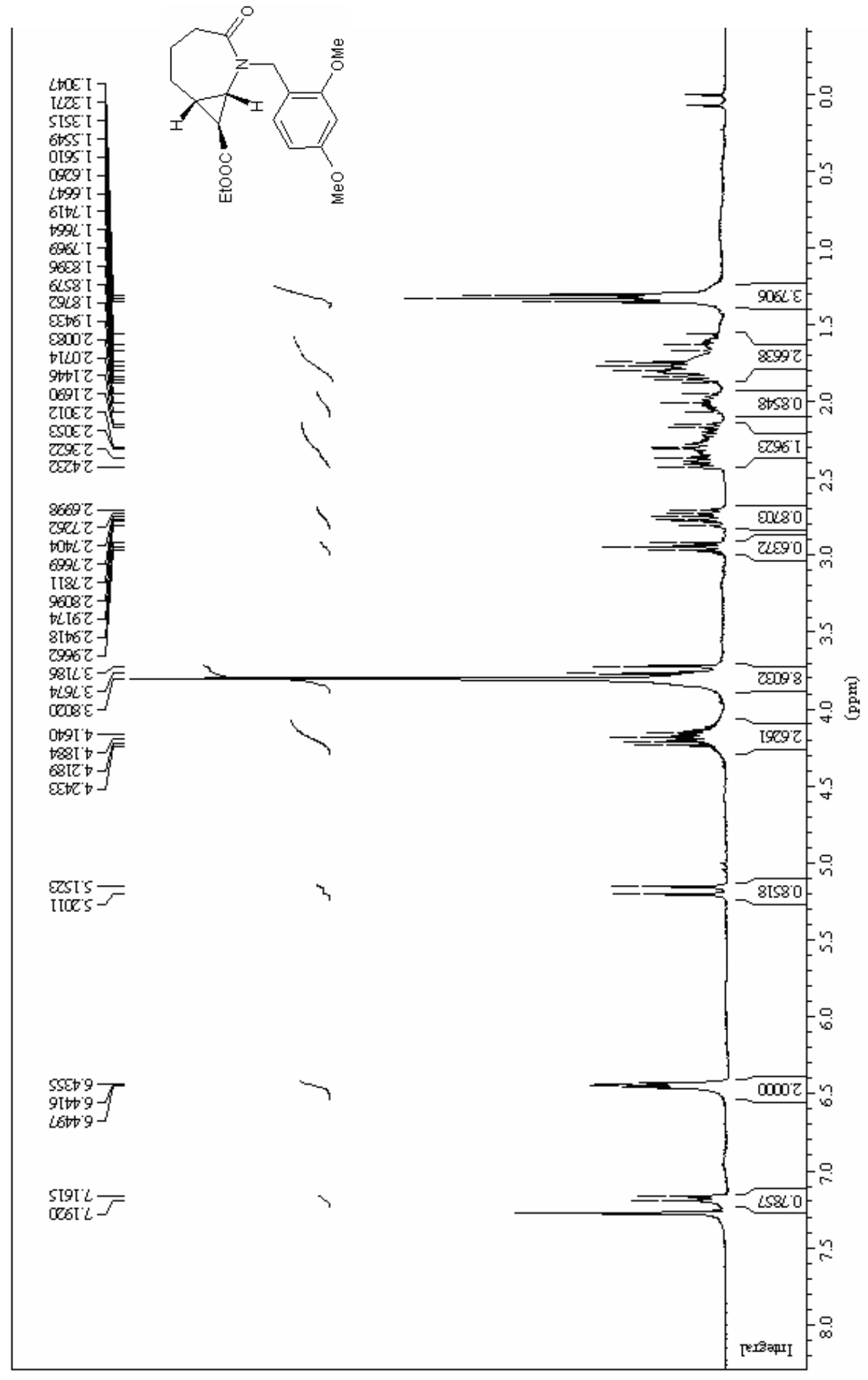




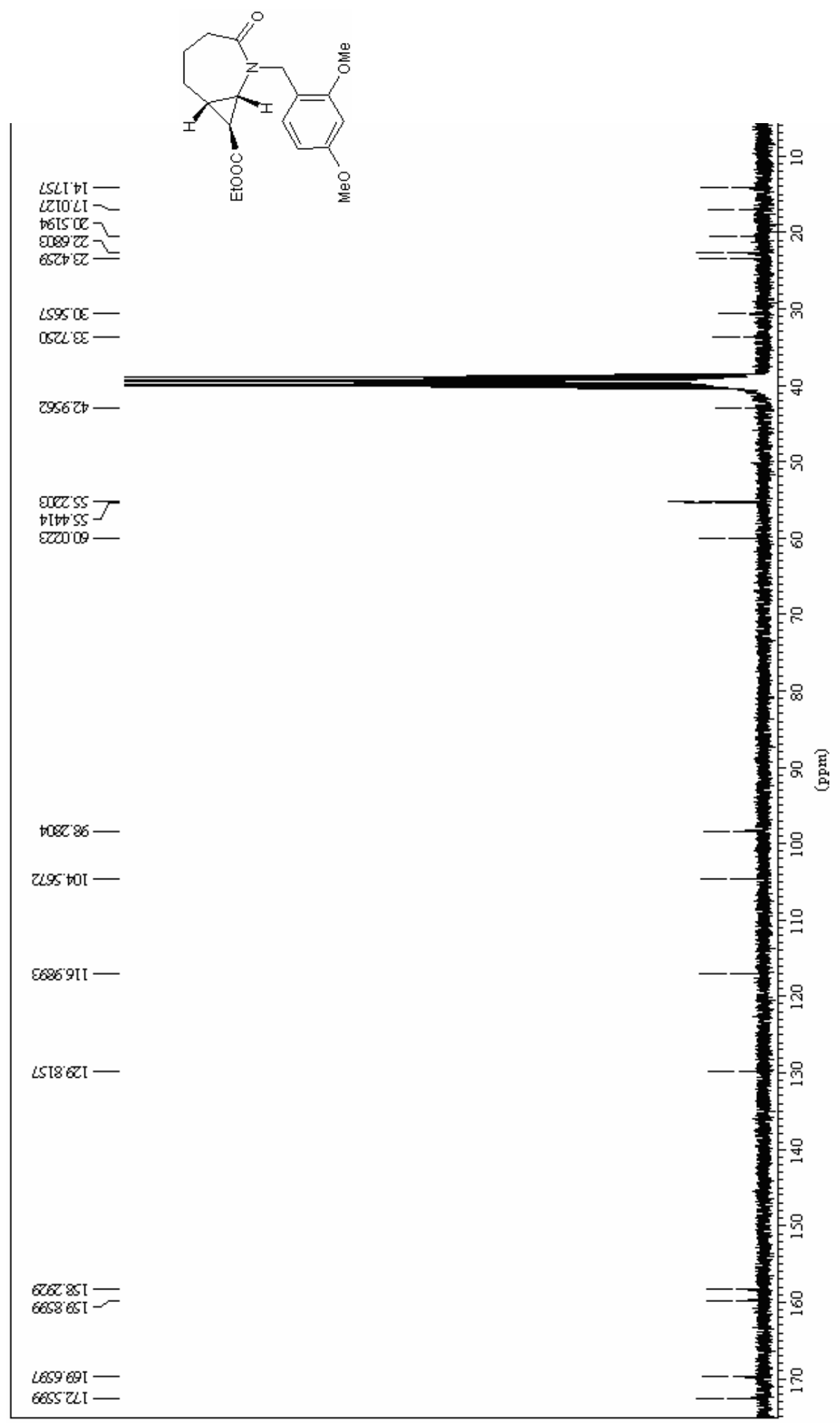


Ethyl $\quad\left(1 R^{*}, 7 S^{*}, 8 R^{*}\right)-4-(2,4-d i m e t h o x y b e n z y l)-3-o x o-4-a z a b i c y c l o[5.1 .0]$ octane-8-carboxylate (18-trans):

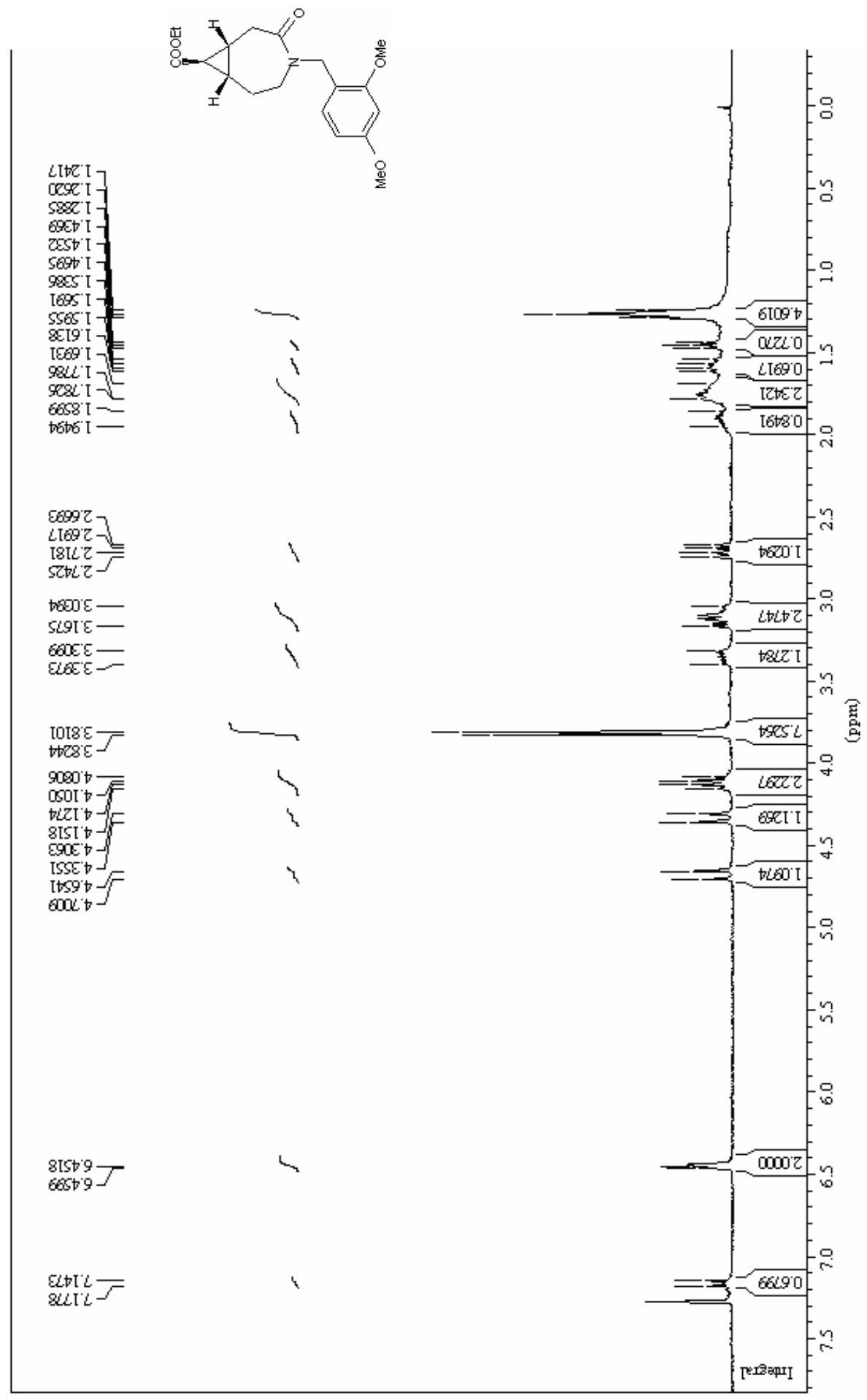




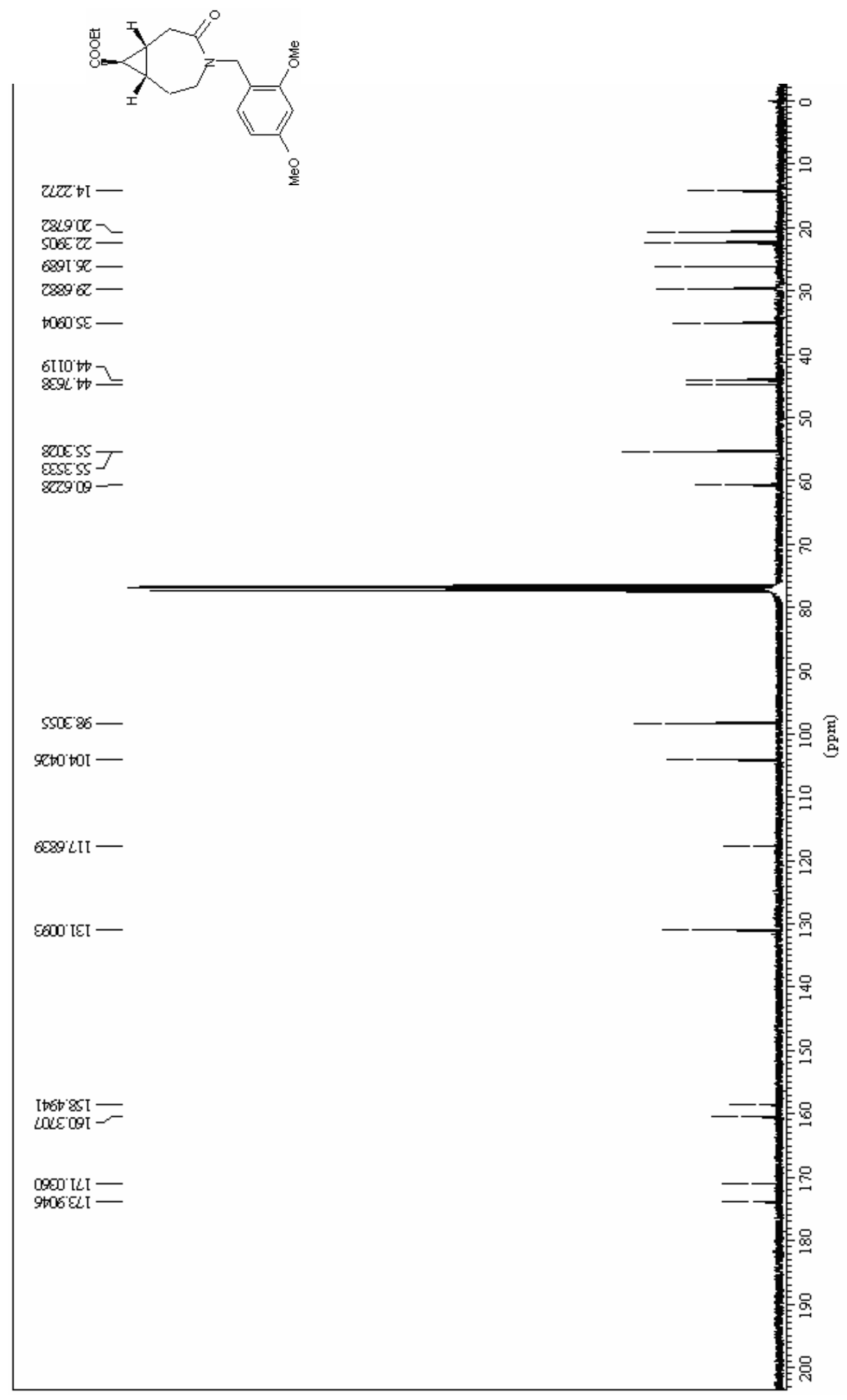


octane-8-carboxylate (19-trans):

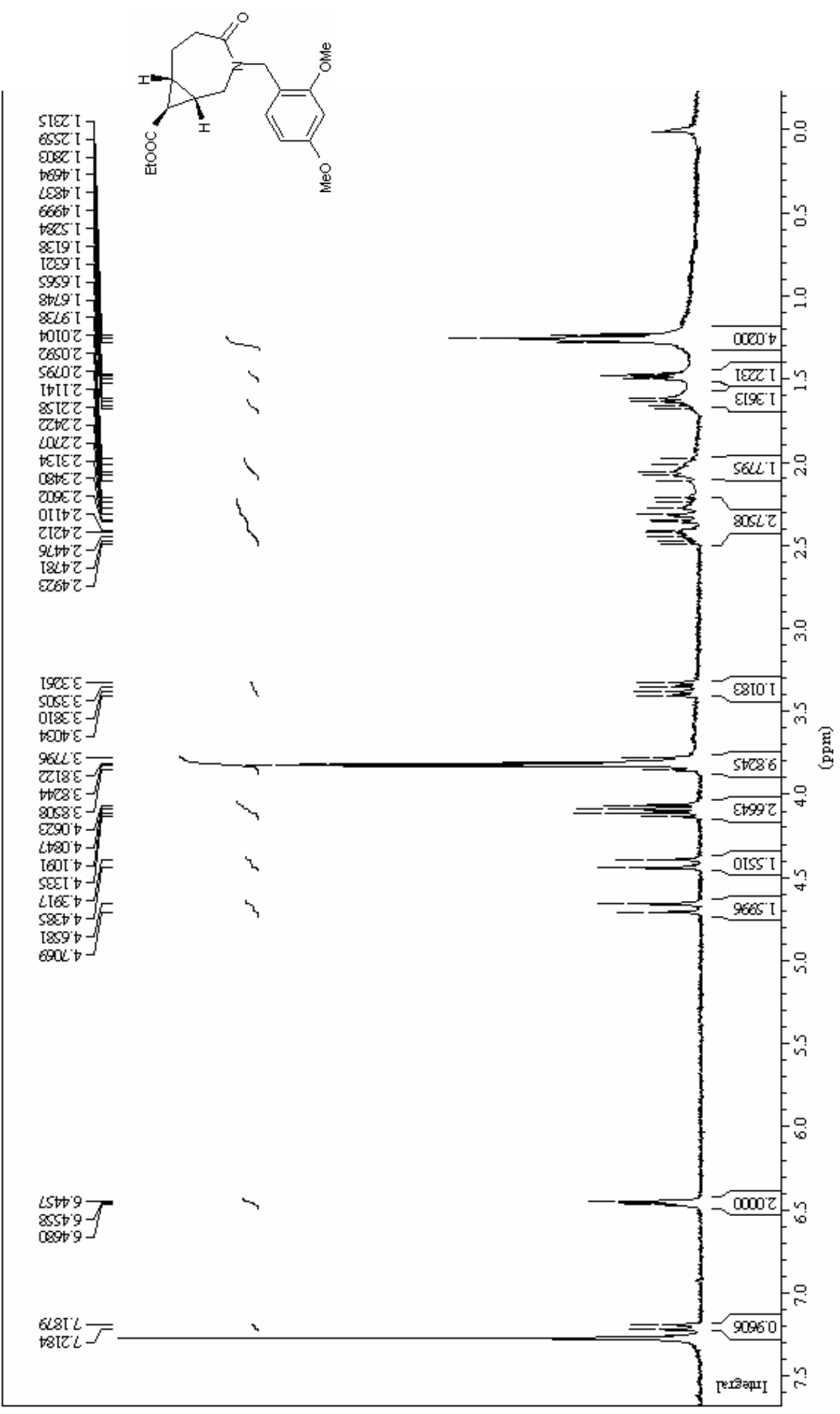




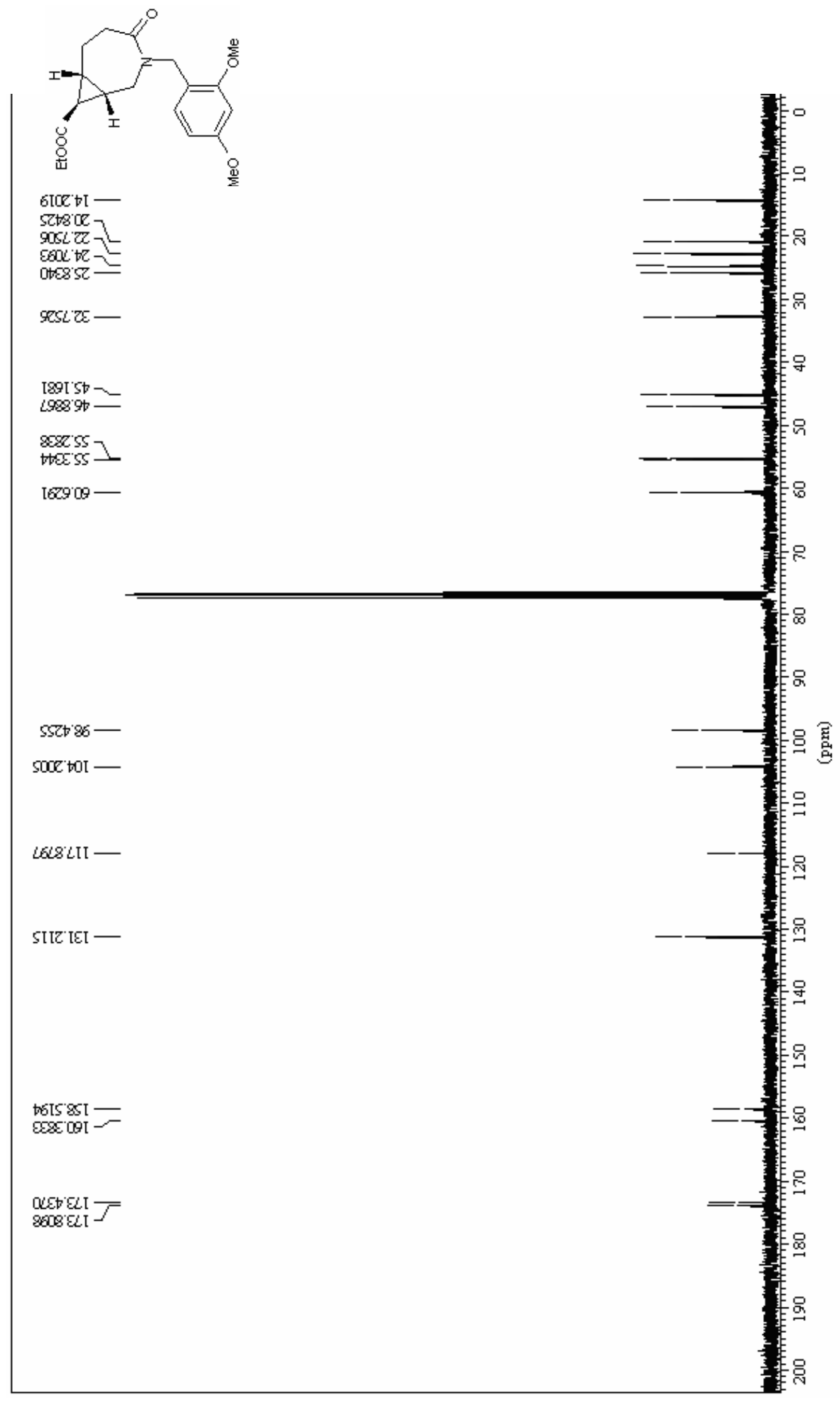

